\title{
CoRoT's view of newly discovered B-star pulsators: results for 358 candidate B pulsators from the initial run's exoplanet field data ${ }^{\star, \star \star}$
}

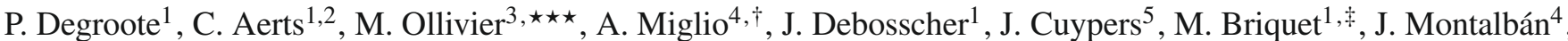 \\ A. Thoul ${ }^{4}$, A. Noels ${ }^{4}$, P. De Cat ${ }^{5}$, L. Balaguer-Núñez ${ }^{6}$, C. Maceroni $^{7}$, I. Ribas $^{8}$, M. Auvergne ${ }^{9}$, A. Baglin ${ }^{9}$, \\ M. Deleuil ${ }^{10}$, W. W. Weiss ${ }^{11}$, L. Jorda ${ }^{10}$, F. Baudin ${ }^{3}$, and R. Samadi ${ }^{9}$ \\ 1 Institute of Astronomy - K.U. Leuven, Celestijnenlaan 200D, 3001 Leuven, Belgium \\ e-mail: pieter . degroote@ster . kuleuven . be \\ 2 Department of Astrophysics, University of Nijmegen, PO Box 9010, 6500 GL Nijmegen, The Netherlands \\ 3 Institut d'Astrophysique Spatiale (IAS), Bâtiment 121, 91405 Orsay Cedex, France \\ ${ }^{4}$ Institut d'Astrophysique et de Géophysique Université de Liège, Allée du 6 Août 17, 4000 Liège, Belgium \\ 5 Koninklijke Sterrenwacht van België, Ringlaan 3, 1180 Brussels, Belgium \\ 6 Departament d'Astronomia i Meteorologia, Universitat de Barcelona, Av. Diagonal, 647, 08028 Barcelona, Spain \\ 7 INAF - Osservatorio di Roma, via Frascati-33, Monteporzio Catone (RM), Italy \\ 8 Institut de Ciències de l'Espai (CSIC-IEEC), Campus UAB, Facultat de Ciències, Torre C5 parell, 2a pl, 08193 Bellaterra, Spain \\ 9 LESIA, UMR8109, Université Pierre et Marie Curie, Université Denis Diderot, Observatoire de Paris, 92195 Meudon Cedex, \\ France \\ 10 LAM, UMR 6110, CNRS/Univ. de Provence, 38 rue F. Joliot-Curie, 13388 Marseille, France \\ 11 Department of Astronomy, University of Vienna, Türkenschanzstrasse 17, 1180 Wien, Austria
}

Received 19 February 2009 / Accepted 26 May 2009

\section{ABSTRACT}

\begin{abstract}
Context. We search for new variable B-type pulsators in the CoRoT data assembled primarily for planet detection, as part of CoRoT's additional programme.

Aims. We aim to explore the properties of newly discovered B-type pulsators from the uninterrupted CoRoT space-based photometry and to compare them with known members of the $\beta$ Cep and slowly pulsating B star (SPB) classes.

Methods. We developed automated data analysis tools that include algorithms for jump correction, light-curve detrending, frequency detection, frequency combination search, and for frequency and period spacing searches.

Results. Besides numerous new, classical, slowly pulsating B stars, we find evidence for a new class of low-amplitude B-type pulsators between the SPB and $\delta$ Sct instability strips, with a very broad range of frequencies and low amplitudes, as well as several slowly pulsating B stars with residual excess power at frequencies typically a factor three above their expected g-mode frequencies. Conclusions. The frequency data we obtained for numerous new B-type pulsators represent an appropriate starting point for further theoretical analyses of these stars, once their effective temperature, gravity, rotation velocity, and abundances will be derived spectroscopically in the framework of an ongoing FLAMES survey at the VLT.
\end{abstract}

Key words. methods: data analysis - stars: oscillations - stars: variables: general

* The CoRoT space mission was developed and is operated by the French space agency CNES, with the participation of ESA's RSSD and Science Programmes, Austria, Belgium, Brazil, Germany, and Spain. All frequency tables, including the identification of combination frequencies, are only available as online material.

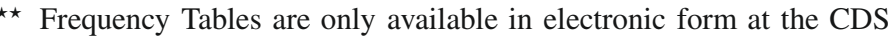
via anonymous ftp to cdsarc.u-strasbg.fr (130.79.128.5) or via

http://cdsweb.u-strasbg.fr/cgi-bin/qcat?J/A+A/506/471

$\star \star \star$ Bâtiment 121, 91405, Orsay Cedex, France.

$\dagger$ Postdoctoral Researcher, Fonds de la Recherche Scientifique FNRS, Belgium.

\# Postdoctoral Fellow of the Fund for Scientific Research, Flanders.

\section{Introduction}

In the forthcoming months and years, the French-European Space mission CoRoT will measure brightness variations of tens of thousands of different stars in its exoplanet programme, with a precision typically two orders of magnitude better than we can achieve from ground-based observations today. This means that we are able to extend the sample of known pulsating stars to class members with fainter apparent magnitude. The study of stellar oscillations from the exoplanet data of the mission is part of the additional programme (Weiss et al. 2004).

In this paper, we focus on CoRoT's initial run in the anticentre direction of the Galaxy (IRa01). We first describe our methods of analysis, as future runs will also be addressed with the same methodology. During IRa01, which lasted some 55 days, the CoRoT satellite was pointed at a field in the galactic plane, towards the galactic anticentre $\left(\alpha \approx 06^{\mathrm{h}} 44^{\mathrm{m}}, \delta \approx-01^{\circ} 12^{\prime}\right)$. We 
Table 1. Overview of the main properties of $\beta$ Cep and SPB pulsators.

\begin{tabular}{rcccc}
\hline \hline Star type & $T_{\text {eff }}(\mathrm{K})$ & Mass $\left(M_{\odot}\right)$ & $f\left(\mathrm{~d}^{-1}\right)$ & Main modes \\
\hline SPB & $10000-22000$ & $3-8$ & $0.3-1$ & $g$ modes \\
$\beta$ Cep & $20000-30000$ & $7-20$ & $4-12$ & $p$ modes \\
\hline
\end{tabular}
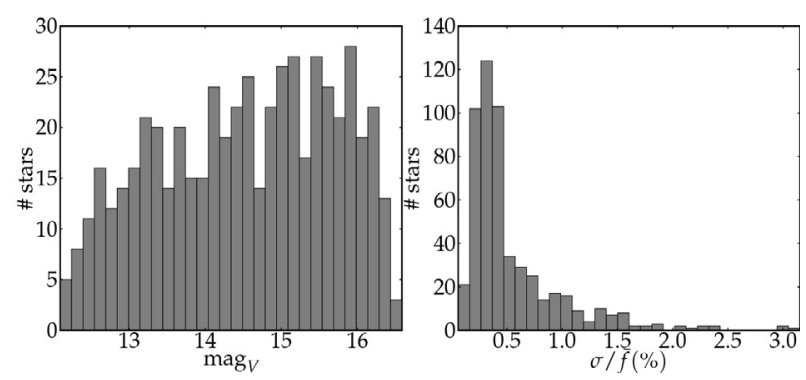

Fig. 1. Distribution of visual magnitudes (left) and the ratio of the light curve's standard deviation to the mean of the flux (right).

are focusing on the $\beta$ Cep stars (e.g. Stankov \& Handler 2005) and slowly pulsating B stars (SPBs, Waelkens 1991) discovered in this field. A short descriptive overview of the main properties of these two classes is listed in Table 1. There is a small overlap between their instability regions, where hybrid pulsators of spectral type near B3, exhibiting both high-order $g$ and loworder $p$ modes, are predicted but not yet observed. On the other hand, these two types of modes have been observed in hotter $\beta$ Cep stars ( $v$ Eri: Handler et al. 2004; 12 Lac: Handler et al. 2006; $\gamma$ Peg: Chapellier et al. 2006). The mechanism that drives the pulsations is the $\kappa$ mechanism (Dziembowski \& Pamiatnykh 1993 ) because of the Fe-group opacity bump at $T \sim 2 \times 10^{5} \mathrm{~K}$, which means that the excitation of modes in those stars is sensitive to the behaviour of opacity in the stellar interior and to the abundance of Fe-group elements.

It would be a huge loss of resources to start analysing all stars in the CoRoT database only to study the B-type pulsators. Therefore, we resort to the results obtained in the framework of the variability classification programme described in Debosscher et al. (2009), hereafter termed CVC, which extracts a basic number of relevant parameters from each light curve to compare them with training sets of stars of known type.

In this paper, we focus on all light curves given a label of either $\beta$ Cep or an SPB star. We also include the $\delta$ Sct and $\gamma$ Doradus stars to limit the red edge of the SPB instability strip and to discuss possible overlap between $\beta$ Cep and $\delta$ Sct stars, on the one hand, and between SPB and $\gamma$ Doradus stars on the other, in terms of light curve properties (e.g., frequency values). In total, 540 light curves are extracted. We emphasise that the results from the CVC classifier are only used to select the initial sample of stars from the CoRoT database.

\section{Description of the dataset and pre-processing}

All datasets are taken over a period of $55 \mathrm{~d}$. Two time samplings occur, one measurement every $\sim 32 \mathrm{~s}$ or one every $\sim 512 \mathrm{~s}$. This sampling is not always constant for one light curve, it may change from low temporal resolution to high temporal resolution during the observing run. The magnitudes of the stars in the sample lie between 12.1 and 16.6 and vary over different scales (Fig. 1).

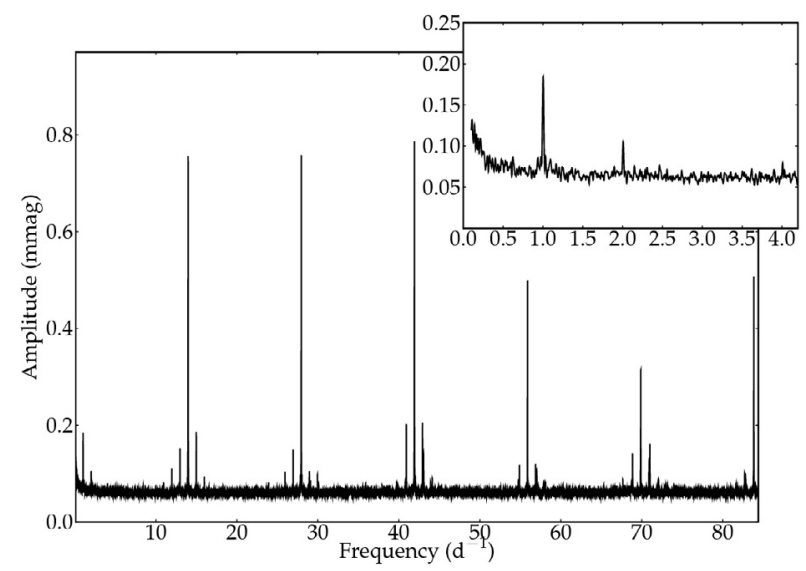

Fig. 2. Averaged Fourier periodogram of 100 non-variable stars. The inset is a zoom on the low-frequency region. A low-frequency powerlaw trend is present, as are the 1 and 2 day alias and the orbital frequency with many of its harmonics and day aliases.

To have an idea of the different kinds of instrumental effects we can expect, as well as their impact on the analysis, we analysed 100 constant stars and averaged their Fourier periodogram. They were selected with the criterion that their dominant frequency has a $p$-value $>0.01$. From Fig. 2 we can identify at least three different groups of features: a low-frequency powerlaw trend due to instrumental drifts (red noise), which becomes negligible around $0.4 \mathrm{~d}^{-1}$, peaks at 1,2 , and $4 \mathrm{~d}^{-1}$ and peaks connected with the orbital frequency due to temperature changes. We refer to Auvergne et al. (2009) for a discussion of the origin of these instrumental peaks and to Samadi et al. (2006) for a description of the CoRoT data pipeline and treatment of data during the passage of the South Atlantic Anomaly.

The most variable feature is the low-frequency powerlaw trend, because of which we introduced a pre-processing step to minimise its potential influence. Two phenomena add up to this trend: discontinuities in the light curve and long-term effects, possibly of instrumental origin. It is impossible to decide whether or not these longterm trends are real variations in the star's light curve, but because we do encounter trends suspiciously linear or exponential, we choose to eliminate them. This also means that we effectively remove possible oscillating behaviour and intrinsic trends on the timescale of the time series itself.

Long-term trends are removed in the following iterative way. First, jumps are detected by sliding two adjacent bins over the lightcurve. Physically, we only expect to find a gradual, continuous rise or decay in the measured flux. To accomodate for the dependence of amplitude and present pulsations, we compare the difference between the average of the bins to the overall difference. If at any point the difference is too high to be considered continuous, as defined by the entire light curve, we identify the point as a discontinuity, perform a piecewise detrending of the two separate parts of the lightcurve and try to detect other jumps. If no more jumps are detected, the piecewise detrending is redone using the original light curve and the discontinuities previously detected. There is some arbitrariness in the choice of parameters (bin width, threshold value etc.), so they were fixed empirically. For the piecewise detrending, the best fit of three simple models was chosen for each part; either a linear, a quadratic, or an exponential trend. An example is provided in Fig. 3. 


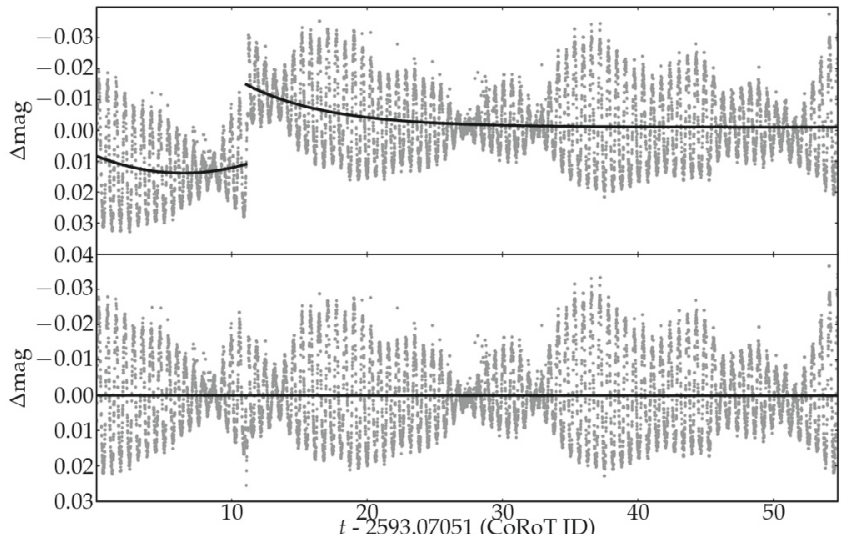

Fig. 3. Correction of one discontinuity in the light curve of SPB candidate 102815542. The first part and second parts were detrended using a quadratic polynomial and exponential decay model, respectively.

\section{Frequency analysis methodology}

\subsection{Basic treatment}

To analyse all jump-corrected and detrended light curves, we describe the flux $F(t)$ as

$F\left(t_{i}\right)=\mu+C+\sum_{i=1}^{n} A_{i} \sin \left(2 \pi\left(f_{i} t+\phi_{i}\right)\right)+\epsilon_{i}$,

where $\epsilon_{i}$ is a Gaussian noise component, $\mu$ the observed average flux level, and $C$ a usually small correction parameter on $\mu$. In this model, there are $n$ nonlinear parameters $f_{i}$, for which the values are estimated using the Lomb-Scargle version of the discrete Fourier transform (Lomb 1976; Scargle 1982). The linear parameters $A_{i}$ and $\phi_{i}$ describe the amplitude and the phase of the corresponding pulsation, respectively, and are estimated by ordinary least squares regression. The error on the frequency determination (and other parameters) is calculated according to Montgomery \& O'Donoghue (1999). These error determinations underestimate the real error because of correlation effects in the data. In our sample, this factor lies between 1 and 4 for the first frequency, and decreases for subsequent frequencies (Schwarzenberg-Czerny 2003).

Traditionally, the frequency acceptance criterion of Breger et al. (1993), using a signal-to-noise ratio (SNR) lower limit of $S N R=4$ in the amplitude periodogram for a peak to be considered genuine, has been proven to be very successful, since very few frequencies identified this way (if at all) had to be refuted afterwards. However, there is no rule about how large one has to choose the interval in the periodogram to use for noise calculation. Second, this method is not suited to detecting regions of power excess, either due to densely packed frequencies or stochastically excited frequencies. Third, related to the previous argument, the SNR method does not guarantee that, if a peak is considered as due to noise, there is no other, possibly even smaller, peak in the periodogram that is not noise. Therefore, the SNR method is not fully objective in its use.

Instead of using the classical SNR criterion, we make an assumption on what the datasets should look like when devoid of instrumental effects and variability from the star itself. A reasonable (yet not perfect) assumption is that we are dealing with white Gaussian noise and that the number of datapoints is large. Degroote et al. (2009) provide an extensive discussion of the deviation from white Gaussian noise and have shown that it can be

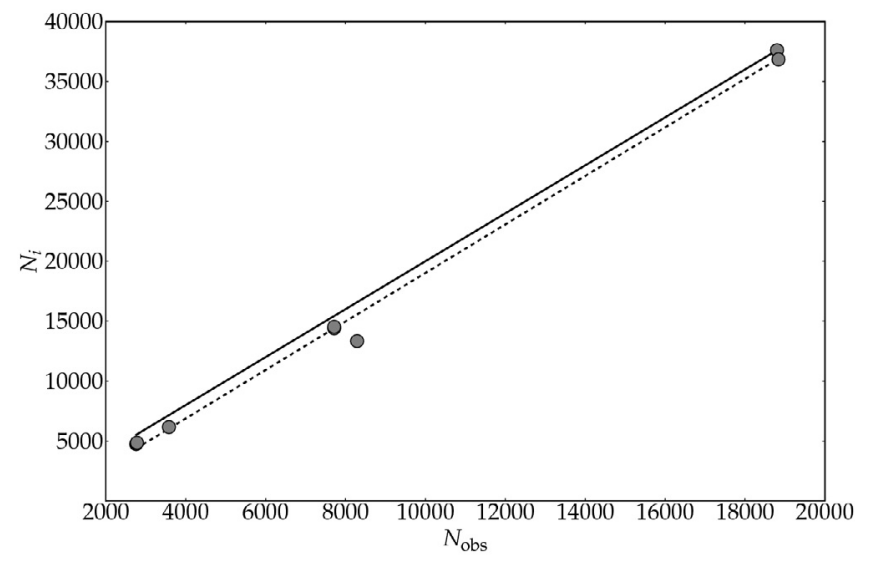

Fig. 4. Result of a simulation study to determine the number of independent frequencies in the CoRoT datasets (sold line: evenly spaced case, $N_{i}=2 N_{\text {obs }}$, dotted line: fit through the simulation results).

ignored in the frequency analysis, as long as a correction factor for correlated data is used. Under this assumption, the distribution of the Scargle power spectrum (normalised with the total variance of the data) belongs to the exponential family (Scargle 1982),

$P(z)=\exp (-z)$,

and thus the noise level is located at a height of $z=1$. In reality, we never test a single frequency, but cover a wide range of possible frequencies, between 0 and the highest detectable frequency, the Nyquist frequency $f_{\mathrm{Ny}}$. In theory, this number is well-defined regardless of the time spacing (Eyer \& Bartholdi 1999). In practice, however, this frequency is usually too high to be of any use (e.g. Koen 2006). A better practical guess for the Nyquist frequency in uneven time sampling is obtained via

$f_{\mathrm{Ny}}=\frac{1}{2 \Delta t}$

with $\Delta t$ in our case the value of the larger timesteps.

To account for the simultaneous testing of all frequencies up to $f_{\mathrm{Ny}}$, we apply the Bonferroni correction (Scargle 1982) to Eq. (2):

$P_{\mathrm{B}}(z)=1-[1-\exp (z)]^{N_{i}}$.

For equally spaced data, theory predicts that the number of independent frequencies $N_{i}$ between 0 en $f_{\mathrm{Ny}}$ scales with the number of observations $N_{\text {obs }}$ as

$N_{i}=2 N_{\text {obs }}$.

In the case of unevenly spaced data, this parameters $N_{i}$ loses its meaning (e.g. Frescura et al. 2008). However, a simulation study shows that expression (5), combined with definition (3), turns out to be a useful and conservative approximation of the true number of independent frequencies. For eight different time templates taken from a CoRoT light curve, we generated 10000 light curves consisting of white Gaussian noise. Next, we calculated the height $z$ of the maximum peak in the Scargle periodogram. These were combined, and a false alarm function of the form (4) fitted through the data, to determine the free parameter $N_{i}$. The result can be seen in Fig. 4, and shows that the CoRoT data allow the use of Eq. (5).

Although the sampling rate is usually much higher than strictly needed, we choose not to rebin the data or to convert the flux to magnitude, because such manipulations alter the shape of a light curve. 


\subsection{Search for combination frequencies}

In particular cases, model (1) can be expanded further to lower the number of dependent parameters and thus simplify the model in terms of $f_{i}$. Neglecting this effect implies imposing a linear model on all observed light curves, thus treating every found pulsation frequency as an independent mode. Identifying dependent frequencies can be seen as a basic correction of the linear model for nonlinear effects:

$$
\begin{aligned}
F(t)=\mu+C & +\sum_{k=1}^{n_{f}} A_{k} \sin \left[2 \pi\left(f_{k} t_{i}+\phi_{k}\right)\right] \\
& +\sum_{l=1}^{m_{f}} A_{l} \sin \left[2 \pi\left(f_{l} t_{i}+\phi_{l}\right)\right]
\end{aligned}
$$

with

$f_{l}=n_{l}^{1} f_{l}^{1}+n_{l}^{2} f_{l}^{2}+n_{l}^{3} f_{l}^{3}$,

where $n_{f}<n$ and $f_{l}$ represent combination frequencies. This might come from nonlinear effects, e.g., nonlinear coupling between modes (Buchler et al. 1997; Handler et al. 2006; Degroote et al. 2009, e.g.). In model (6), the linear parameters still have to be fitted, but are sometimes expected to be in phase or in antiphase with each other. In contrast to the window frequencies, combination frequencies and harmonics are not artificially introduced, because they have a real physical interpretation. It is thus important to derive them.

The search for combination frequencies is done in the following way: for each frequency, the harmonics are identified using all empirically found frequencies with higher amplitude than the one under investigation. Those are then excluded from the list of independent frequencies. For the remaining frequencies, all second and third order combinations are tested, again using only the frequencies with higher amplitude, and not part of a combination so far (in order to avoid higher order "combinations of combinations"). To keep the parameter space manageable, frequencies of order $n$ are only considered if at least one of order $(n-1)$ is previously identified. We are limited by the total time of observations, so there is some ambiguity in the choice of possible deviation from the exact combination. Because the Rayleigh limit of $L_{\mathrm{R}}=1 / T$ is a natural measure of maximum uncertainty on the frequency determination, we consider this a reasonable value to test the presence of combinations.

\subsection{Search for period and frequency spacings}

The variability detected in SPB stars is interpreted as stemming from low degree, high-order modes whose periods are known to be sensitive to the structure of the stellar core. The first-order asymptotic approximation developed by Tassoul (1980) shows that the period spacing $(\Delta P)$ between modes of consecutive order and same degree is constant and can be approximated as

$\Delta P=2 \pi\left(L \int_{x_{0}}^{1} \frac{N}{x} \mathrm{~d} x\right)^{-1}$,

where $L=[\ell(\ell+1)]^{1 / 2}$ (with $\ell$ the mode degree), $x$ the normalized radius, $x_{0}$ corresponds to the boundary of the convective core and $N$ the Brunt-Väisälä frequency. Moreover, as shown by Miglio et al. (2008), deviations from a uniform period spacing are very sensitive probes of the chemical composition gradient in the star that develops near the edge of the convective core. A sound detection of period spacing in SPB stars would therefore

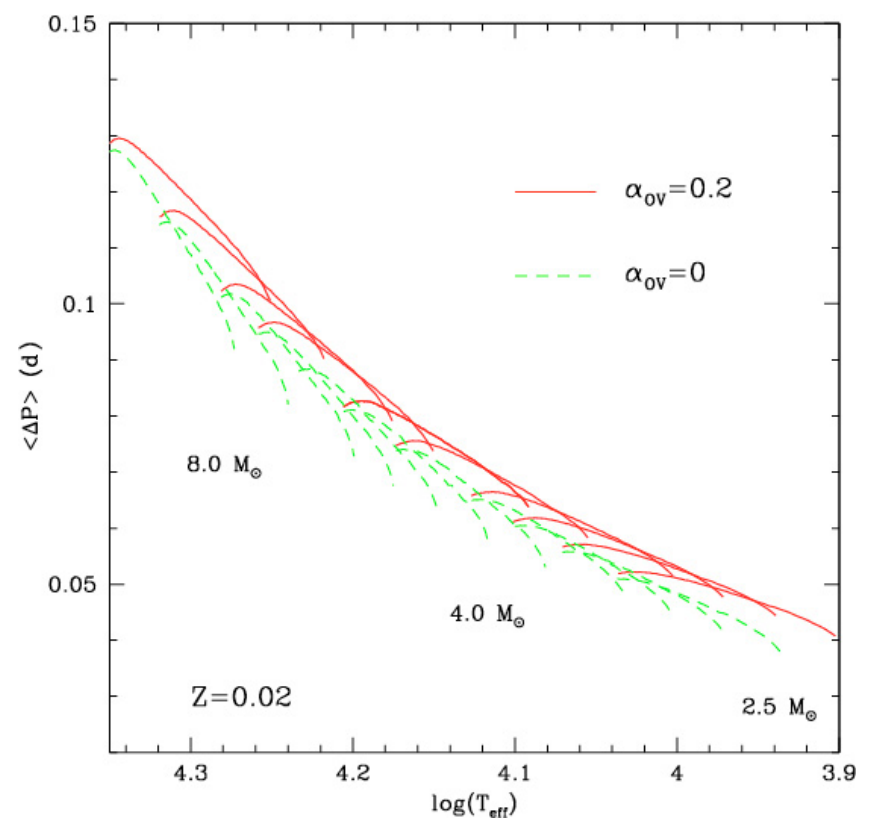

Fig. 5. Theoretically predicted asymptotic $\ell=1$ period spacing of highorder $g$ modes $(\Delta P)$ as a function of the effective temperature. $\Delta P$ is computed for main-sequence models with masses between 2.5 and $8 M_{\odot}$, with (full line) and without (dashed line) overshooting from the convective core. The heavy-element mass fraction assumed in the models is $Z=0.02$.

allow direct inferences on the near-core structure of B stars. A theoretical estimate of the expected average $\Delta P$ for $\ell=1$ modes is reported in Fig. 5, where main-sequence models between 2.5 and $8 M_{\odot}$, computed with and without overshooting, are considered. We have to recall, however, that such a regular period spacing could be strongly affected by the effects of rotation on such long oscillation periods (see e.g. Dziembowski et al. 1993 for a discussion).

Analogously to the case of g-mode periods, a regular frequency spacing can occur for $p$ modes. Other situations where a form of spacing can be found, is in frequency multiplets originating from stellar rotation, where deviations from the equidistance case can also occur. A systematic search for recurrent frequency/period spacings therefore represents a valuable tool for a theoretical interpretation of the large number and variety of pulsating stars detected by CoRoT. The total time span of 55 days for the Initial Run puts an upper limit of $\sim 0.02 \mathrm{~d}^{-1}$ on the frequency resolution. For rich SPB pulsators, the frequencies are expected to be densely packed, thus detecting period spacings may be difficult.

To detect spacings in a list of frequencies $\left(f_{1}, \ldots, f_{n}\right)$ or periods $\left(P_{1}, \ldots, P_{n}\right)$, we use the Kolmogorov-Smirnov test of Kawaler (1988) and the inverse variance test of O'Donoghue (1994). In addition, a third new test is proposed here. For the latter, we first construct a vector containing all possible differences between the periods. This way, we are left with a vector of length $\operatorname{Binom}(n, 2)$, and elements $\Delta_{\mathrm{ij}}=\left|P_{i}-P_{j}\right|$. For a test spacing $\Delta P$, we calculate for each element of these entries $\Delta_{i j}$,

$r=\Delta_{\mathrm{ij}}-\left\lfloor\frac{\Delta_{i j}}{\Delta P}\right\rfloor \Delta P$,

where $\lfloor x\rfloor$ is the greatest integer smaller than $x$. We count the number $s_{o}$ of elements in the intervals

$s_{o} \rightarrow[0, \epsilon] \cup[\Delta P-\epsilon, \Delta P]$. 
This number $s$ captures the observed extreme values, which are defined through the customizable tolerance parameter $\epsilon$. If we assume that these differences are uniformly distributed, we can express the expected number $s_{\mathrm{e}}$ of extreme values by

$s_{\mathrm{e}}=\frac{2 \epsilon}{\Delta P} n_{i}$

Application of the plain matrix test implies computation of the $p$ value according to

$P\left(X_{i} \in[0, \epsilon] \cup[\Delta P-\epsilon, \Delta P]\right)=\frac{\epsilon}{\Delta P}$.

This test is appropriate if some periods are missing or not detected. However, we may prefer to only take those pairs into account for which the difference between the two members is exactly $\Delta P$, and not a multiple. This we can do using the distinct number test. It works the same as the matrix test, except that we keep track of the term

$m=\left\lfloor\frac{\Delta_{i j}}{\Delta P}\right\rfloor \Delta P$

from Eq. (8). Only those spacings where $m=1$ and $r<\epsilon$ or where $m=0$ and $\Delta P-r<\epsilon$ are considered as valid periodspacing candidates. The possible disadvantage of using this test is that we can end up with a lot of periods belonging to the same $\Delta P$, but where no single triplet is detected. Of course, this can be an advantage as well, e.g., in the search for rotational splittings; the matrix distinct number test will detect doublets with the same spacing, far apart around different frequencies. Finally, if we are interested in the number of periods belonging to each test spacing $\Delta P$, we can simply count the number of different periods belonging to each test spacing period set.

To illustrate the performance of these tests, we simulate a collection of periods, in which we have put 3 different spacings (in arbitrary units): $\Delta P_{1}=0.1315$ (8 periods), $\Delta P_{2}=0.4332$ (6 periods), and $\Delta P_{3}=0.3579$ (3 periods). On top of that, we added 8 random periods, to arrive at a total number of 25 periods. We additionally allowed each period to deviate from the rigid spacing according to a random normal fluctuation of $\sigma=$ 0.0005 . The results of the different tests are shown in Fig. 6: the Kolmogorov-Smirnov test locates only the most obvious period spacing, but suffers from a strong bifurcation artifact, already noticed by Kawaler (1988). The inverse variance test shows lack of power in this dense pack of periods; it is only able to detect a spacing of $\Delta P_{1} / 2$. This contrasts to the matrix tests; their statistic is almost devoid of noise, and only show peaks at the true period spacings and their multiples. The matrix test and distinct number test show great similarity in their peak structure, because they have the same basic calculation method. The reason the matrix test favours the larger spacing $\Delta P_{2}$, although it has fewer members than $\Delta P_{1}$, is how we set up the statistic: we gave larger spacings less chance of happening.

Regardless of the succes of these tests, we must be careful in interpreting the results: because of the occurrence of other random periods, we may by chance pick up other pairs of periods with the same spacing, but totally unrelated.

\section{Evaluation of the new pulsators}

\subsection{Additional Strömgren photometry}

To determine the effective temperature $T_{\text {eff }}$ and gravity $\log g$ of the stars in the initial run, Strömgren photometry was obtained using the wide field camera attached to the Isaac Newton

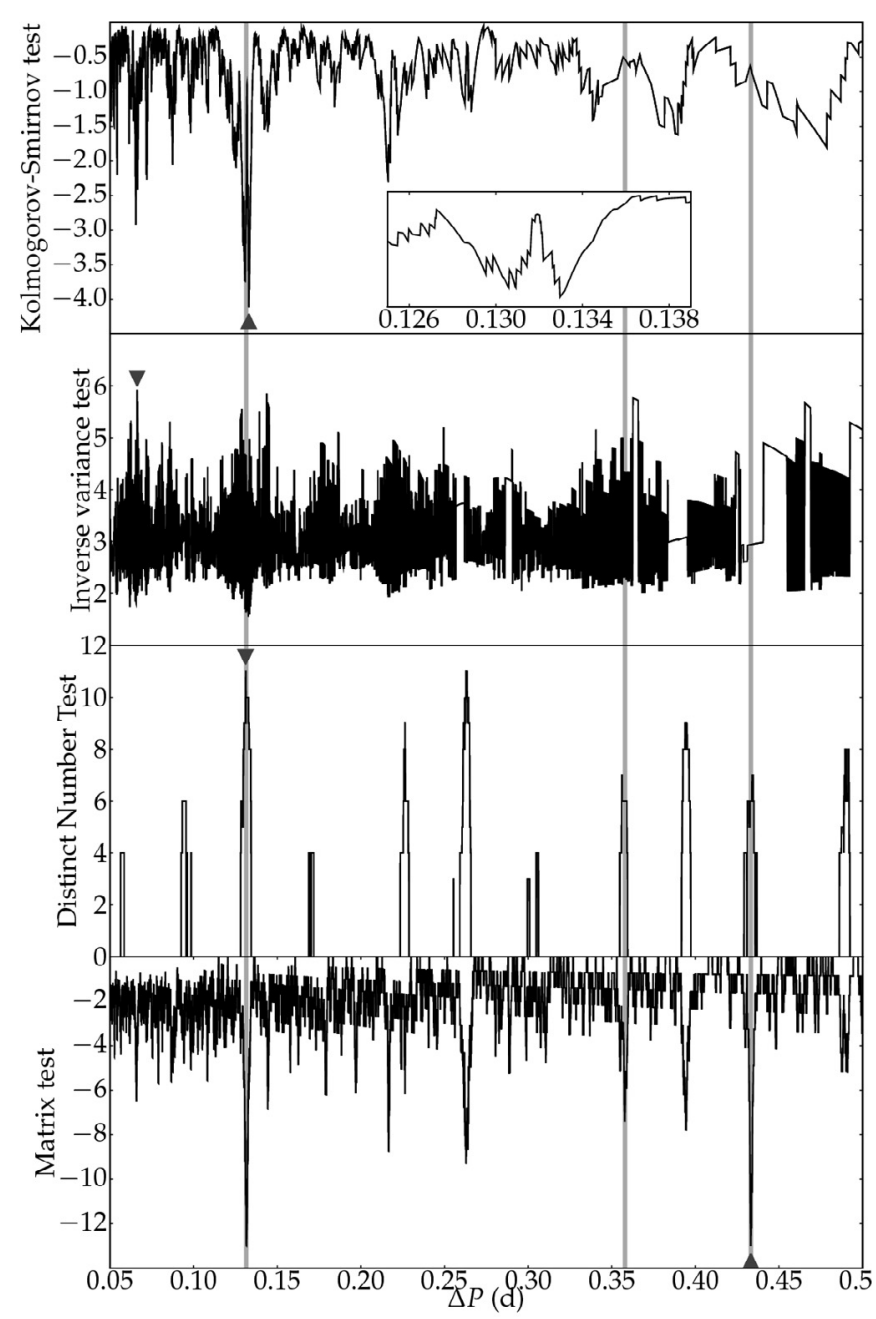

Fig. 6. Outcome of different tests for period spacings on a simulated set of periods. Input spacings $\Delta P_{i}$ and detected spacings $\Delta P_{i}^{\prime}$ were equal for $\Delta P_{1}=0.1315$ and $\Delta P_{2}=0.4332$. For the third spacing $\Delta P_{3}=0.3579$, the detected spacing was $\Delta P_{3}=0.3576$. The (top panel) Kolmogorov-Smirnov test with the typical bifurcation, and inverse variance test (second panel) do not detect all spacings. The distinct number test (third panel) and matrix test (bottom panel) are more suitable to detecting the spacings.

Telescope at the La Palma observatory, Spain. Because of time constraints, no $\beta$ index measurements could be obtained. This implied that we did not have a direct measurement of the gravity. We thus proceeded in an iterative way. First, we computed the dereddened photometric indices by using an appropriate spectral type for each of the pulsators: $\mathrm{B} 2 \mathrm{~V}$ for the candidate $\beta \mathrm{Cep}$ stars, B5V for the candidate SPBs, A2V for the $\delta$ Sct candidates, and F2V for the $\gamma$ Dor candidates. Next, we used $c_{0}$ to predict the $\beta$ index following the relation between these two quantities derived by Balona (1994) for main sequence stars. In fact, Balona (1994) has shown that this procedure has advantages over using the measured indices to compute $\beta$, due to imperfections in the calibrations. With these dereddened indices and $\beta$ estimates, the effective temperatures and gravities were subsequently estimated, using Balona's (1994) calibration.

The procedure described above delivers an estimate of $T_{\text {eff }}$ and $\log g$ with an internal error, i.e., an error assuming that the dereddened input colours are error-free. It is well known, however, that this procedure for determining the fundamental parameters suffers from systematic uncertainties connected with the 


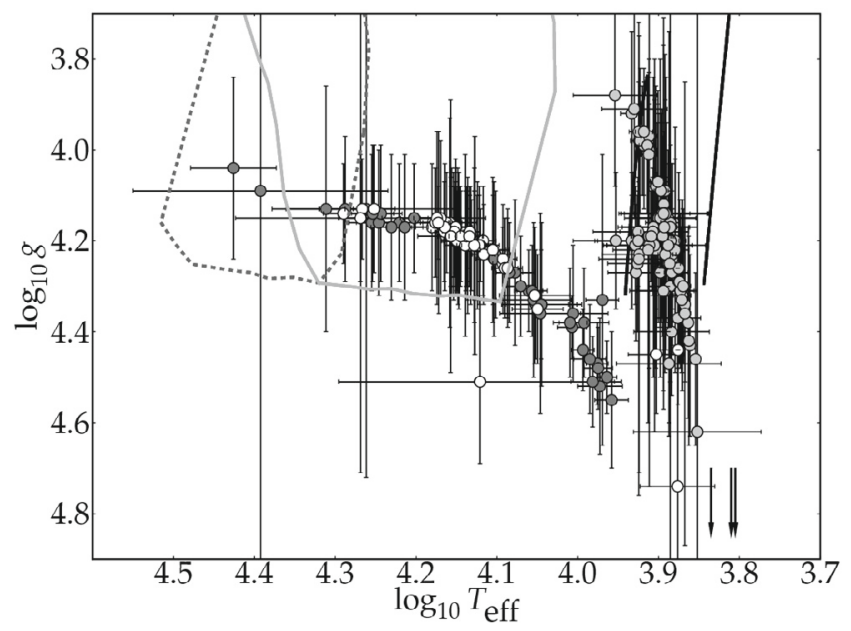

Fig. 7. Location of targets in a $\left(T_{\text {eff }}, \log g\right)$ diagram. The SPB star candidates (white dots, 66 stars) occupy the theoretical instability strips well (grey dashed lines), except for a few outliers (see text). The same holds for the $92 \delta$ Sct candidates (light grey dots), but not for the $43 \beta$ Cep candidates (dark grey dots). Arrows denote outliers: the cool outlier CoRoT 102818325 from the $\beta$ Cep candidates is probably a binary. The two cool outliers on the $\delta$ Sct side each have only two detected frequencies: CoRoT 102921009 has $f_{1} \approx 8 \mathrm{~d}^{-1}, f_{2}=f_{1} / 2$ (possible a binary), and CoRoT 102787451 has $f_{1} \approx 17.4 \mathrm{~d}^{-1}, f_{2} \approx 16.5 \mathrm{~d}^{-1}$.

limitations of the model grids used to explain the behaviour of standard stars. To get a handle on these systematic errors, we also used the calibrations of Moon \& Dworetsky (1985), which are valid along the entire main sequence as well. The standard deviation between the values for $T_{\text {eff }}$ and $\log g$ derived from Balona's (1994) and Moon \& Dworetsky's (1985) calibrations was considered to be a good approximation of the systematic uncertainties, so these were added to the statistical errors.

As a test of the validity of the obtained fundamental parameters for the new B-type candidate pulsators, we placed all stars in the HRD and compared their position with the instability strips (Fig. 7). The numerous new $\delta$ Sct stars match the classical instability strip perfectly, which gives us confidence that the estimates of $T_{\text {eff }}$ and $\log g$ obtained from our procedure are appropriate.

From Fig. 7 we see that the classification is very powerful for extracting candidate new pulsators from a large sample on the basis of white-light photometry alone (as done in Debosscher et al. (2009)), but it is not perfectly reliable on the scale of individual stars. Moreover, we do not expect so many candidate $\beta$ Cep stars compared to SPBs and $\delta$ Sct stars, considering the mass-dependence of the initial mass function and of mainsequence lifetimes: this implies that several of them might have a different character. We therefore decided to regroup all new candidate B stars in the sample, by using the $T_{\text {eff }}$ and $\log g$ information, along with the obtained frequency spectra. This additional classification should allow a better distinction between the classes of pulsators along the main sequence than just using the white-light photometry alone, since we add information on the fundamental parameters. The new clusters we obtain in this way are discussed by means of typical and atypical examples, some of which are shown in the text, while others are reported in the electronic Appendix A.

\subsection{Candidate pulsators situated between the SPB- and $\delta$ Sct instability strips}

The most obvious result from Fig. 7 is undoubtedly the appearance of p-mode type variations (in terms of frequency values), not only far from the theoretical $\beta$ Cep instability strip, but also outside any instability strip. Part of this discrepancy can be attributed to the CVC not containing classes of spotted or differentially rotating stars, or ellipsoidal variables. Unavoidably, the main frequency overlaps with the $\beta$ Cep pulsation range for some of these stars. Moreover, many hot stars in our sample turn out to be good Be star candidates (Neiner, private communication). These are or will be studied and published elsewhere (Gutiérrez-Soto et al. 2008; Emilio et al., in prep., and future papers).

The only remaining $\beta$ Cep candidate is CoRoT 102813271 , where two frequencies are detected in the typical p-mode frequency regime: $f_{1} \approx 5.79 \mathrm{~d}^{-1}, f_{2} \approx 5.51 \mathrm{~d}^{-1}$ and a third frequency $f_{3} \approx 0.23 \mathrm{~d}^{-1}$ in the g-mode regime. Because of its low amplitude and long period, the g-mode frequency can possibly be influenced by instrumental effects.

The objects on the red side of the instability strips do not constitute one uniform group. That they all vary over the same timescale implies that the CVC classified them as $\beta$ Cep stars. However, looking beyond the first few frequencies reveals their distinct characteristics: CoRoT 102833548, 102848985, 102922479, and to a lesser extent also the almost constant star CoRoT 102850576, have a well-structured frequency spectrum consisting of equally spaced frequencies (Fig. A.2 in the Appendix). The results of the spacing tests and a time-frequency analysis of the detrended light curves are shown in Figs. 8 and 9, and indicate that a spacing of $\sim 0.70 \mathrm{~d}^{-1}$ is common. The amplitude of the main peaks are of the order of $0.1 \%$. The frequency range overlaps that of $\beta$ Cep stars on the low side, but also reaches lower frequencies. The determined temperatures place all these stars consistently between the SPB and $\delta$ Sct instability strips.

CoRoT 102861067, 102790063, 102790331, 102729531, 102862454 and 102771057 constitute a second well-separated group. They share a complex frequency spectrum containing between $\sim 20$ and $\sim 170$ significant peaks spread over a wide range and amplitudes below $0.1 \%$. Two examples are shown in Figs. A.5 and A.4, while schematic oscillation spectra are shown in Fig. A.1 in the Appendix. Dense "forests" of closely spaced peaks are identified around some frequencies. Based on the 55 days of photometric observations alone, it is hard to make the distinction between real multiplets and non-stationarity of the amplitudes.

Close to the $\delta$ Sct instability strip, there is another small group of multiperiodic variables: CoRoT 102933855 , 102703484, 102850502, and 102816758. Although these stars have a rich frequency spectrum, they are different from the previous group because their spectrum shows more isolated frequencies with higher amplitudes $(\sim 0.5 \%)$. Schematic oscillation spectra are provided in Fig. A.3.

Near the border of the SPB instability strip, four monoperiodic variables are visible: CoRoT 102921797, 102774512, 102889144, and 102872474. The distinction between monoperiodic oscillation and spots is difficult to make without colour or spectral information.

The main power of all of the above stars is located in the expected $\beta$ Cep pulsation range. However, some of the stars on the red side of the SPB instability strip show variability on longer time scales $\left(<4 \mathrm{~d}^{-1}\right)$, e.g. Figures A.6 and A.7 in Appendix A. 

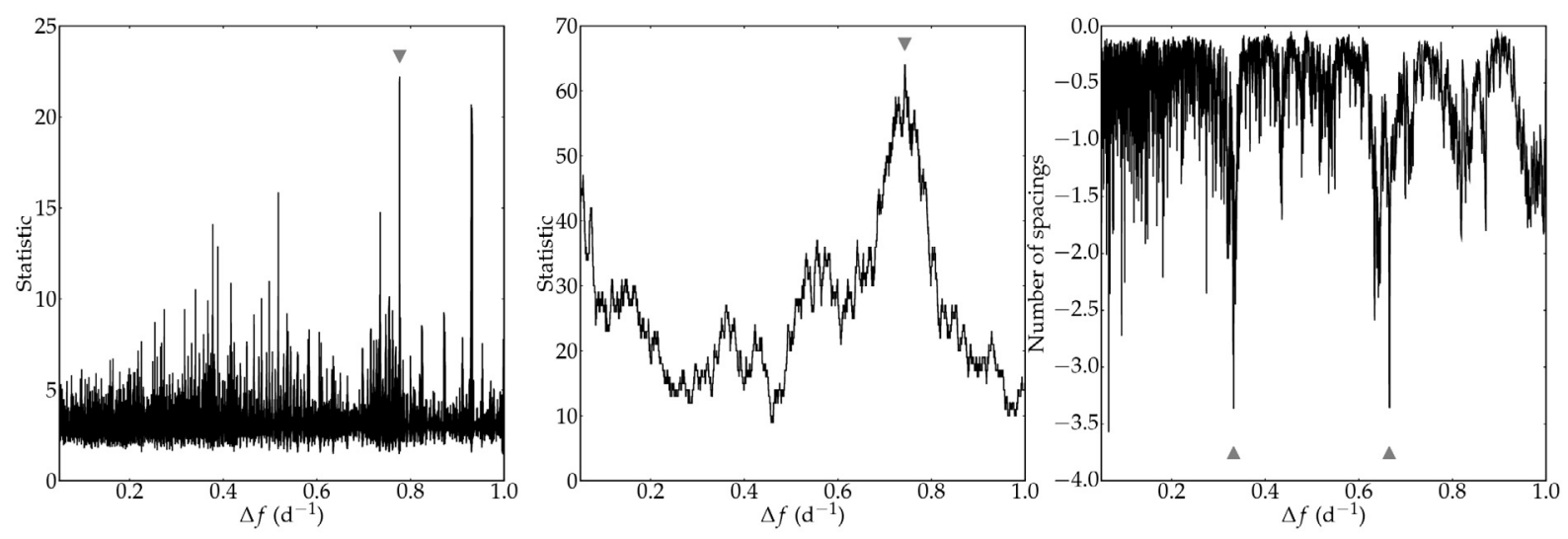

Fig. 8. Selection of spacing test results: (left) the inverse variance test of detected frequencies in CoRoT 102922479 indicates a spacing of $0.78 \mathrm{~d}^{-1}$. The matrix number (middle) test gives a clear spacing of $0.74 \mathrm{~d}^{-1}$ in CoRoT 102848985 . The Kolmogorov-Smirnov (right) test applied to CoRoT 102833548 reveals a spacing of $\Delta P_{1}=0.66 \mathrm{~d}^{-1}$, but does not exclude $\Delta P_{1} / 2$ as an alternative possibility.
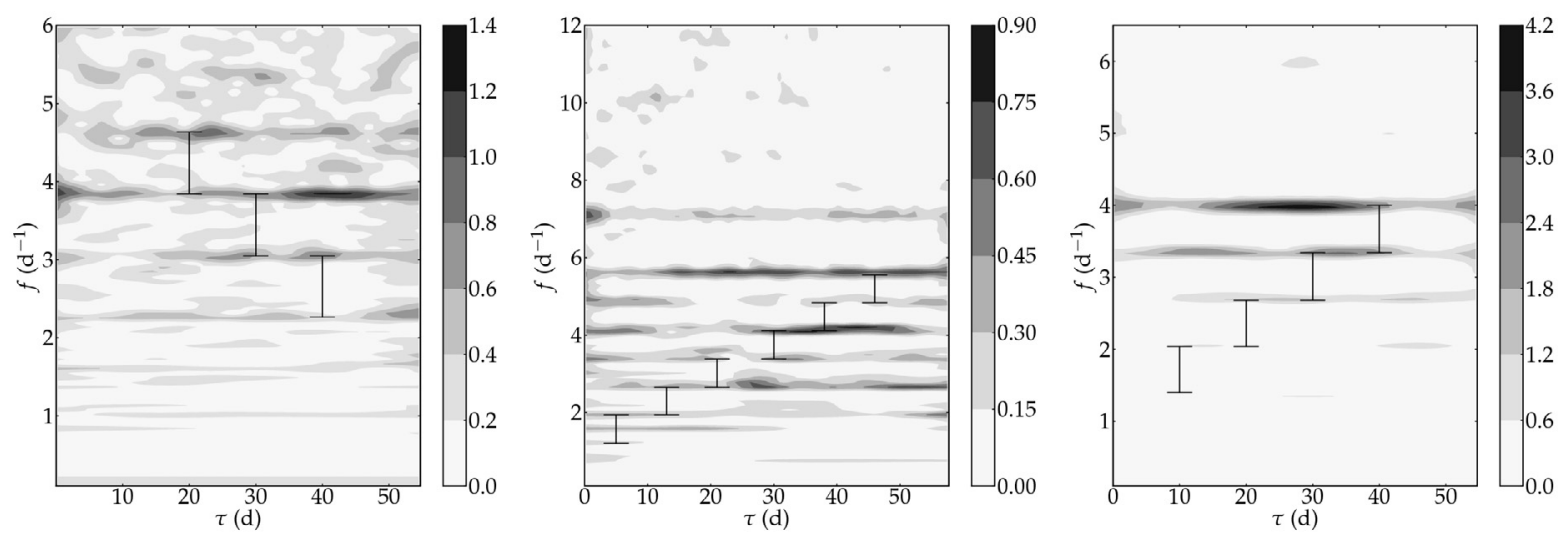

Fig. 9. Time-frequency analysis of CoRoT 102822479 (left), 102848985 (middle), and 102833548 (right). The spacings found (see Fig. 8) and their derived frequency members are depicted by the horizontal short lines, vertically connected.

Not all of this periodic variability is sinusoidal or even approximately sinusoidal. The best example in this respect is CoRoT 102762284 , for which the sinusoidal variation is superposed on a larger scale outburst-like variation (Fig. A.8).

A summary of the interpretation of the cool, short-term (i.e. $\beta$ Cep-like) candidate pulsators in the $\log T_{\text {eff }}-\log g$ diagram is shown in Fig. 12.

\subsection{SPB candidate examples}

As is clear from Fig. 7, the SPB star candidates from the initial classification agree with their instability strip. The 15 clearest SPB candidates, in order of decreasing amplitude, from $\sim 1 \%$ to $\sim 0.2 \%$, are CoRoT 102844894, 102754851, 102728830, 102855391, 102739246, 102797587, 102938439, 102769848, 102848506, 102848506, 102956197, 102943966, 102968674 and 102871668 . Their effective temperature and gravity locates them well within the theoretical SPB instability strip. Besides the typical closely spaced $\mathrm{g}$ mode pulsations, we also find a residual power excess bump in a frequency band above $1.5-2 \mathrm{~d}^{-1}$ for the majority of these stars, well separated from the typical SPB pulsation range predicted for excited modes of $\ell<3$ modes. The theoretical instability strip reaches the observed range of frequencies for modes with a higher $\ell$ value, but at the same time, disk-averaging effects reduce the observed amplitudes. For $\ell=4$, this reduction factor amounts to about 30 , which is still above the CoRoT noise level for many of the SPB candidates, so we cannot exclude the higher observed frequencies being due to high-degree modes. We illustrate the nature of the observed variability for a prototype of these stars (CoRoT 102938439) in Figs. 14, 15, and 13. Other examples can be found in Figs. A.9 to A.12). After prewhitening all low frequencies, the largest feature in the amplitude spectrum turns out to be a collection of closely spaced peaks (Fig. 15). At least two phenomena can cause these features: unresolved stable modes with a complex beating pattern, or time-dependent oscillation frequencies and/or amplitudes. In the first case, we expect a region with blended or nearly blended frequency peaks, without any amplitude structure. In the second case, we expect a frequency region with a peak at the excited frequency, and Lorentzian shape farther away from the central peak. When a residual light curve of these stars is constructed by prewhitening a nonlinear least squares fit of all frequencies below $\sim 1.5 \mathrm{~d}^{-1}$, the same amplitude structure emerges for all of them, which points towards the same physical origin given that the pattern is not chaotic. The amplitudes of the detected modes are highly variable over time (see Figs. A.9 and A.10). The observed features could come from modes with a finite lifetime or to nonlinear nonresonant distortion leading to time-dependent phenomena. An analysis of the evolution of the amplitudes shows that the period over which the modes 


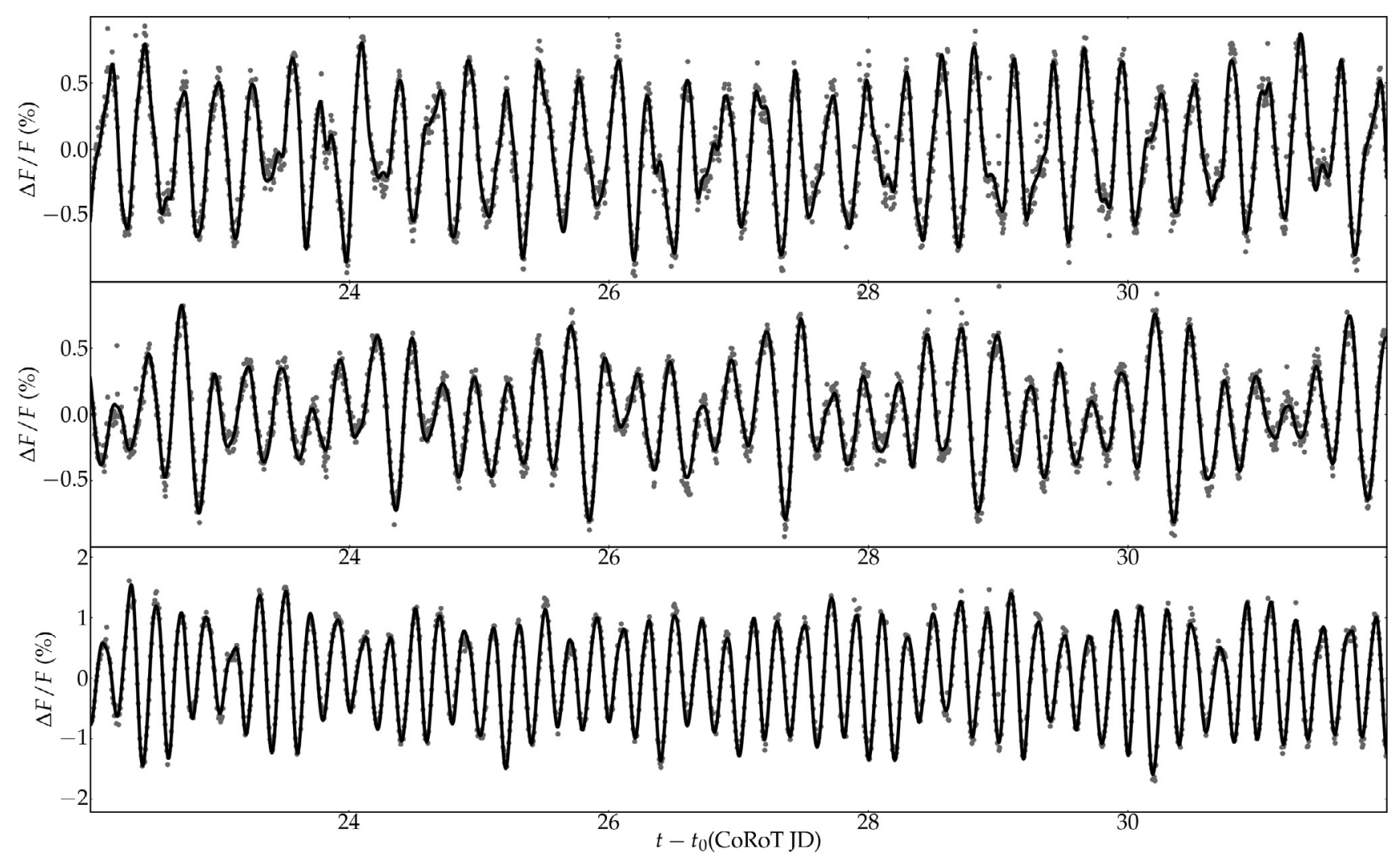

Fig. 10. B-type pulsator candidates on the red side of the SPB instability strip. Some of these stars show clear, stable oscillations. (top) CoRoT $102816758\left(t_{0}=2593.0705\right.$ CJD, $\left.f_{1} \approx 3.6 \mathrm{~d}^{-1}\right)$, (middle) CoRoT $102833548\left(t_{0}=2593.0705\right.$ CJD, $\left.f_{1} \approx 4.0 \mathrm{~d}^{-1}\right),($ bottom $)$ CoRoT $102850502\left(t_{0}=2593.0705 \mathrm{CJD}, f_{1} \approx 5.0 \mathrm{~d}^{-1}\right)$. Light curves with high time resolution were binned per $\sim 10$ datapoints for visibility reasons. The black line represents the fit from which the satellite orbital frequency, its harmonics, and its aliases were filtered out.

appear and dissappear is of the order of one to a few days. Instead of a sinusoidal shape, the light curve maxima are narrower and sharper, while the minima are shallow and broad (Fig. 13). This is very similar to the light curves of some white dwarf pulsators and is explained by nonlinear effects for these objects (Vuille et al. 2000). We notice that a similar feature of disappearing and reappearing frequencies was also found in the Be star HD 49330 and was interpreted as the cause of an outburst (Huat et al. 2009). It could very well be that this is a general phenomenon at low amplitudes for B stars, but that it only leads to mass loss for the most rapid rotators among them.

A second manifestation of nonlinearity can be found in many detected combination frequencies. In contrast to the timedependent phenomenon described above, these are stable on the scale of the total time span, thus resulting in isolated frequency peaks in the spectra (see online frequency tables). A detailed description of these modes is difficult based on the short time span of the Initial Run and the long periods of these modes $\left(<2 \mathrm{~d}^{-1}\right)$, but will surely be possible for similar new pulsators in the CoRoT long run data.

Secondary good SPB star candidates include CoRoT 102863407, 102752912, 102773435, 102917802, 102826973, 102838201, 102888003, 102915048, 102813396, and 102945383 of which the last four also show evidence for modes of finite lifetime.

\subsection{Other variable objects}

Brightness variations need not necessarily be due to pulsations (alone). For example, when two binaries are physically close to each other and the stars are of comparable luminosity and size, and the grazing eclipses can resemble one single, stable sinusoidal pulsation. A DFT analysis puts more power in the double frequency of the orbital frequency, folding both the primary and secondary eclipse onto each other. Therefore, close binaries with an orbital period roughly between 0.25 and $0.5 \mathrm{~d}^{-1}$ can easily be mistaken for a $\beta$ Cep star, whereas a wider system can be misinterpreted as an SPB. Examples of close or contact binaries are CoRoT 102725806, 102858055, 102876625, 102930503, 102784048, 102768686, 102732139, 102873761 , and 102812372. The phase diagram of the first candidate, CoRoT 102725806, is shown in Fig. 11. We find an orbital frequency of $f=2.925(7) \mathrm{d}^{-1}$, independently confirmed with the phase dispersion minimization and information entropy method. The amplitude of the primary eclipse is $A_{1}=8.56 \%$, compared to the average flux level. The double system was mistaken for a single $\beta$ Cep star by the CVC because of the small difference between the amplitude of the primary and secondary eclipse; $A_{1}-A_{2}=0.87 \%$. The duration of the primary eclipse in phase units is $P_{1} \approx 0.08$, which is almost equal to the duration of the secondary eclipse, $P_{2} \approx 0.10$.

The possibility of a binary system and an SPB candidate are not mutually exclusive, because CoRoT 102918586, 102912741 


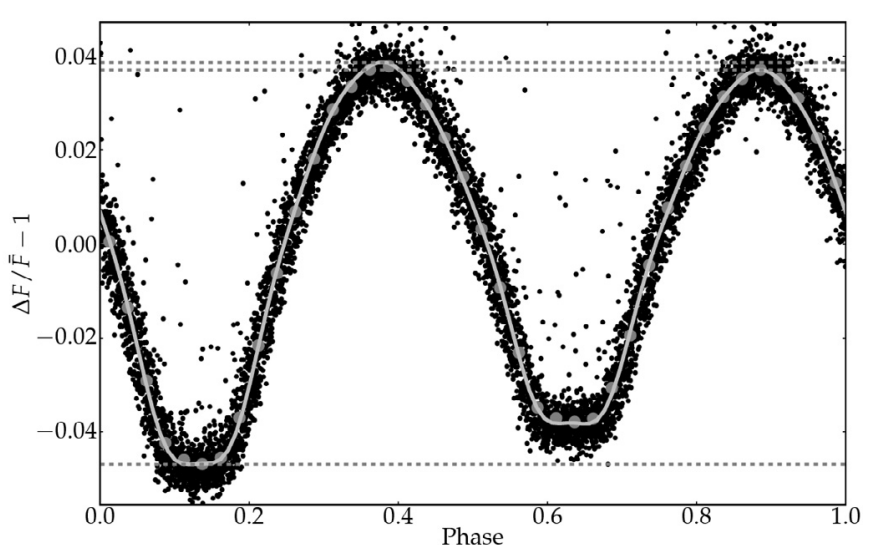

Fig. 11. Binary system CoRoT 102725806 with an orbital frequency $f=2.925(7) \mathrm{d}^{-1}$. Black circles are data points, dark grey circles are phase-binned data points, the light grey line is a fit using 7 harmonics, determined via consecutive prewhitening.

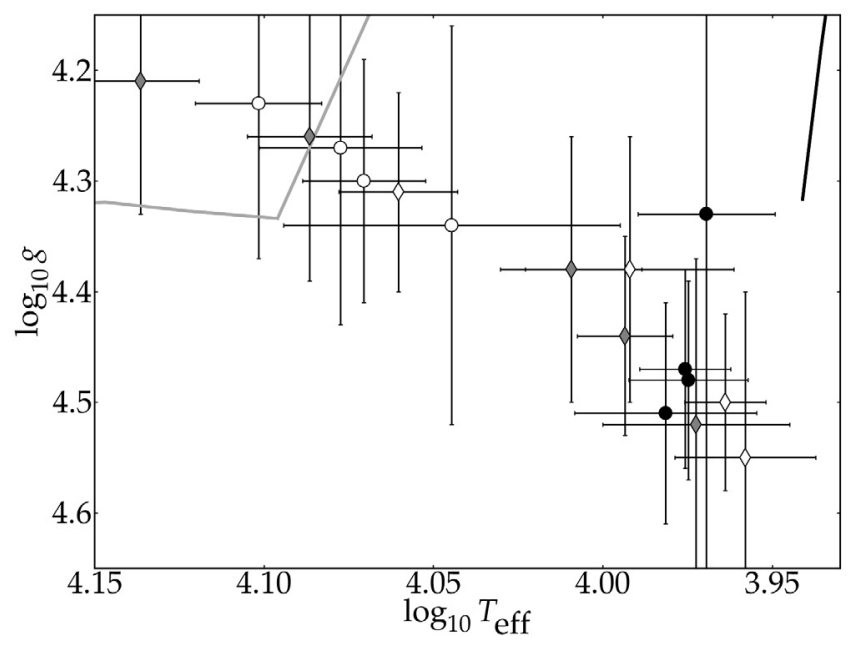

Fig. 12. Location of $\beta$ Cep candidate pulsators in the $\log T_{\text {eff }}-\log g$ diagram with a determined $T_{\text {eff }}$ too low to be a real $\beta$ Cep star: $\delta$ Sct like variables (black dots), monoperiodic $\beta$ Cep candidates (white dots), rich pulsators (grey diamonds), and targets with equidistant frequency spacing (black white diamonds).

and CoRoT 102793963 turn out to be candidate SPBs in close binaries, of which the latter shows a single sinusoidal variation, synchronised with the orbit of the binary. These systems in particular can be of interest for asteroseismology, because the binary orbit can help to constrain the mass and radius of the SPB star.

Another group of SPB candidates have frequencies in a very broad range, e.g. CoRoT 10282445, 102764403, 102818535, 102816987, 102837646, 102804931, 102887852, 102930369, 102790135, and 102856178. CoRoT 10282445, for example, shows periodic variations with time scales around $\sim 1 \mathrm{~d}^{-1}, \sim 4 \mathrm{~d}^{-1}$ and $\sim 15 \mathrm{~d}^{-1}$ (Fig. 16). The main frequency $f_{1}=1.0411(7) \mathrm{d}^{-1}$ can be connected to the third frequency $f_{3}=1.1533(3) \mathrm{d}^{-1}$, resulting in a beating period of almost 10 days. The second frequency $f_{2}=15.271(9)$ is connected to $f_{29}=14.739(4) \mathrm{d}^{-1}$, and the same spacing of $0.52 \mathrm{~d}^{-1}$ is found throughout the whole frequency spectrum (Table A.1). Although the spacing connects the three main frequency regions where variability occurs, current

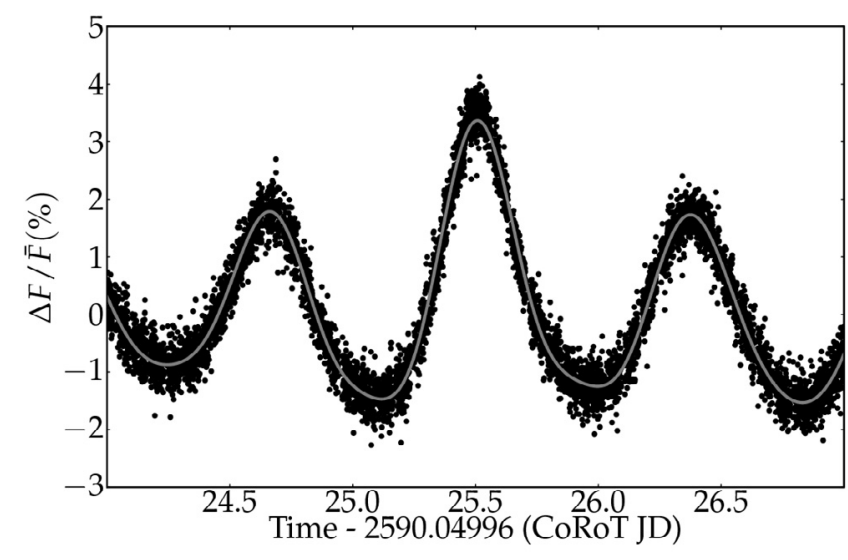

Fig. 13. CoRoT 102938439: excerpt of original light curve and fit (solid grey line), around a time where the amplitude is temporarily driven to a higher level. The typical shape of shallow minima and sharp maxima is visible.

stellar models do not offer any interpretation in terms of a single $\mathrm{SPB}, \beta \mathrm{Cep}$, or $\delta$ Sct star, while we can exclude contamination from nearby stars on the CCD. The possibility of a binary system consisting of a hybrid $\beta$ Cep and a $\delta$ Sct remains, as well as the one of a triple system. Spectra will help us to unravel the exact cause of the variation in this light curve.

For a couple of light curves (CoRoT 102910610, 102968526, 102857565, and 102876625, see Fig. 17), spot models seem more appropriate for describing them than a pulsation model; the resemblance to simulated light curves of rotating spotted stars is indeed apparent (e.g. Strassmeier \& Bopp 1992; Lanza et al. 1993). Their frequency spectra agree with those models, and few frequencies are detected in a very narrow interval. Figure 18 illustrates that it can be difficult to distinguish simple beating patterns from spot related variability. By assuming that the star rotates differentially like the Sun, the multiplet structure can be used to determine the level of differential rotation $\Delta \Omega$, e.g., for CoRoT $102968526, \Delta \Omega=0.0538 \pm 0.0002 \mathrm{~d}^{-1}$. This implies that one part of the star completes one more full rotation cycle over a period of $1 / \Delta \Omega$.

\section{Comparison with theoretical predictions}

The results presented in the previous section show that many of the stars classified as an SPB or $\beta$ Cep star by the CVC classifier are not typical members of the two classes (at least as far as the classes have been defined so far). To have a general comparison between observed and theoretically predicted instability domains, we not only consider $\log T_{\text {eff }}-\log g$ planes (see Fig. 7), but also confront predicted and observed frequency instability domains, as presented in Fig. 19.

The theoretical instability domains were computed for nonrotating main-sequence models of masses between 1.2 and $18 M_{\odot}$ and 2 values of the heavy-elements mass fraction: $Z=$ 0.02 and $Z=0.01$ (respectively upper and lower panels of Fig. 19). Stellar models were computed with CLES (Scuflaire et al. 2008b), adiabatic frequencies with LOSC (Scuflaire et al. 2008a), and the stability of modes of degree $\ell=0,1,2$ was investigated with the code MAD (Dupret et al. 2003). In the case of $\delta$ Sct and $\gamma$ Doradus stars, the nonadiabatic computations included the interaction between convection and pulsation as described by Grigahcène et al. (2005). All stellar models were computed with overshooting from the convective core 


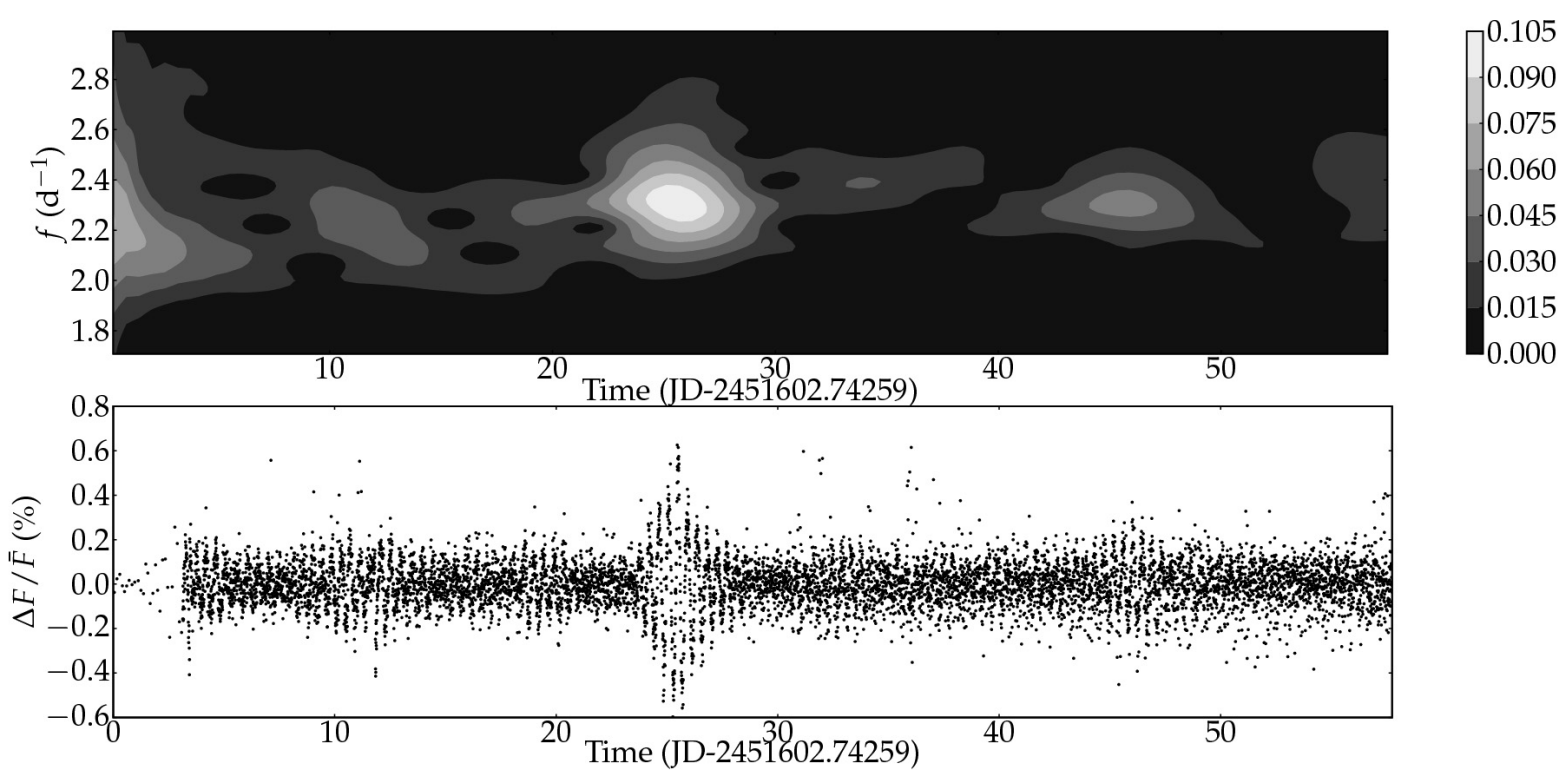

Fig. 14. (Top panel) Short Time Fourier Transformation of the residual light curve of CoRoT 102938439, after prewhitening a nonlinear fit with all detected significant frequencies below $\sim 1.5 \mathrm{~d}^{-1}$. The highest amplitude frequencies in the residuals clearly show modulated amplitudes. (Bottom panel) A binned version of the residual lightcurve clearly shows the largest temporal variability near day 25 of the light curve, with some smaller "outbursts" as well.

$\left(\alpha_{\mathrm{OV}}=0.2\right)$ and a mixing-length parameter $\alpha_{\mathrm{MLT}}=2.0$, both expressed in local-pressure, scale-height units. OP opacity tables (Badnell et al. 2005) with Asplund et al. (2005) metal mixture were adopted. We refer to Miglio et al. (2007a,b) and Zdravkov \& Pamyatnykh (2008) for a detailed study of the effect of considering different opacity tables and metal mixtures on the excitation of pulsation modes in B-type stars. The distinct islands of instability populating the $\log T_{\text {eff }}$-Period diagram of Figs. 19 correspond (from left to right) to $\beta$ Cep-, SPB-, $\delta$ Sct-, and $\gamma$ Dor-type pulsations.

Stars classified as SPBs are in overall agreement with the theoretically expected domain (see Figs. 7 and 19), though in some cases with frequencies higher than those of a typical SPB star (which could be due to the strong effects of rotation on the periods of oscillation). On the other hand, several stars classified as $\beta$ Cep are in the typical $T_{\text {eff }}$ instability domain of SPBs. These stars have frequency peaks in the $\beta$ Cep frequency domain: their modes could be prograde SPB-type modes as proposed in, e.g., Saio et al. (2007).

The effects of rotation may also affect the determination of $T_{\text {eff }}$ for a few stars that have a frequency spectrum resembling the one of fast-rotating SPBs but that, based on their position in Fig. 19, are cooler than the red edge of the SPB instability strip (e.g. CoRoT 102833548 and 102729531). A more reliable spectroscopic $T_{\text {eff }}$ determination of these stars is thus needed to clarify the nature of these targets. Such spectroscopic data will become available in the next months.

The comparison with theoretical expectations will also greatly benefit from the determination of the number of pulsating B stars relative to all the B stars observed in CoRoT's IRa01 EXOfield. The absence of typical $\beta$ Cep pulsators (in terms at the same time of frequency spectrum and $T_{\text {eff }}$ ) can be ascribed simply to the expected scarcity of early B-type stars in the field, following the initial mass function and stellar evolution models. This requires the determination of stellar parameters for a large number of stars and will be addressed in a future work.

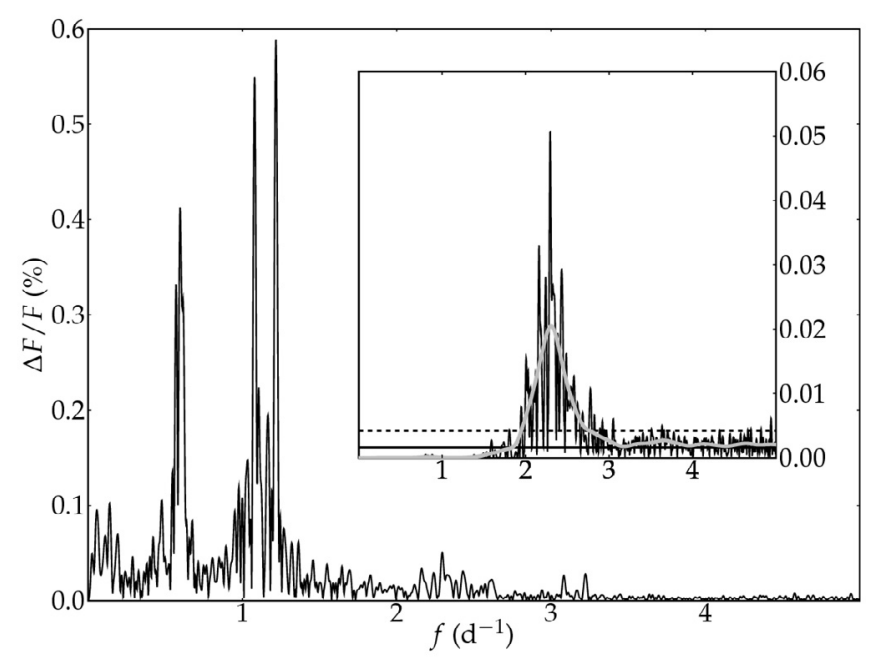

Fig. 15. Original periodogram of CoRoT 102938439. The inset is the amplitude spectrum of the residuals, after prewhitening all significant frequencies below $\sim 1.5 \mathrm{~d}^{-1}$. Grey solid line is a Gaussian smoothing with $\sigma=0.075 \mathrm{~d}^{-1}$. The black solid line denotes the mean noise level, the dotted line is the $99 \%$ significance level.

It is clear from these first general remarks that the relevance of CoRoT's photometric variability survey goes beyond the simple definition of instability domains of expected and known types of pulsators. Particular theoretical interest is provided by the unexpected variability detected in several targets on the red side of the SPB instability strip, a region where several claims of the detection of pulsations were made (the so-called "Maia" stars, see e.g. Scholz et al. 1998; Aerts \& Kolenberg 2005). The theoretical instability mechanisms proposed as an explanation for these stars do not cover the full area in the HertzsprungRussell diagram where such pulsations have been claimed (see Townsend 2005 \& Savonije 2005). The forthcoming spectroscopic observations of those targets will shed new light on these 

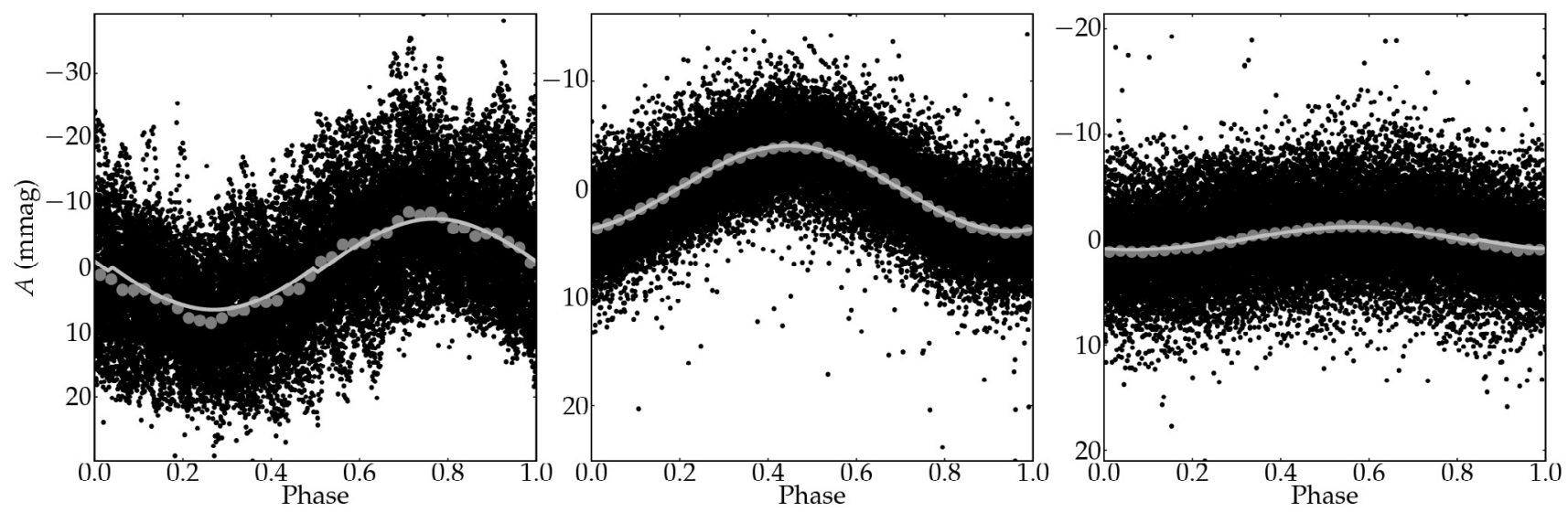

Fig. 16. Phase diagrams of three clear sinusoidal variations of CoRoT 102804522 . The frequency in the left panel $\left(f \approx 1.04 \mathrm{~d}^{-1}\right)$ is rather high for typical $g$ modes in SPBs, while the frequency in the middle panel $\left(f \approx 15.27 \mathrm{~d}^{-1}\right)$ is rather high for a typical $\beta$ Cep star $p$-mode, but quite typical for $p$ modes in $\delta$ Sct stars. The frequency with which the light curve is folded in the right panel $\left(f \approx 4.05 \mathrm{~d}^{-1}\right)$ could, on the other hand, be a typical $p$ mode in a $\beta$ Cep star. This different behaviour raises the suspicion that this is not one single star, although there are neither traces of eclipses nor any indication of flux contamination from nearby stars on the CCD.

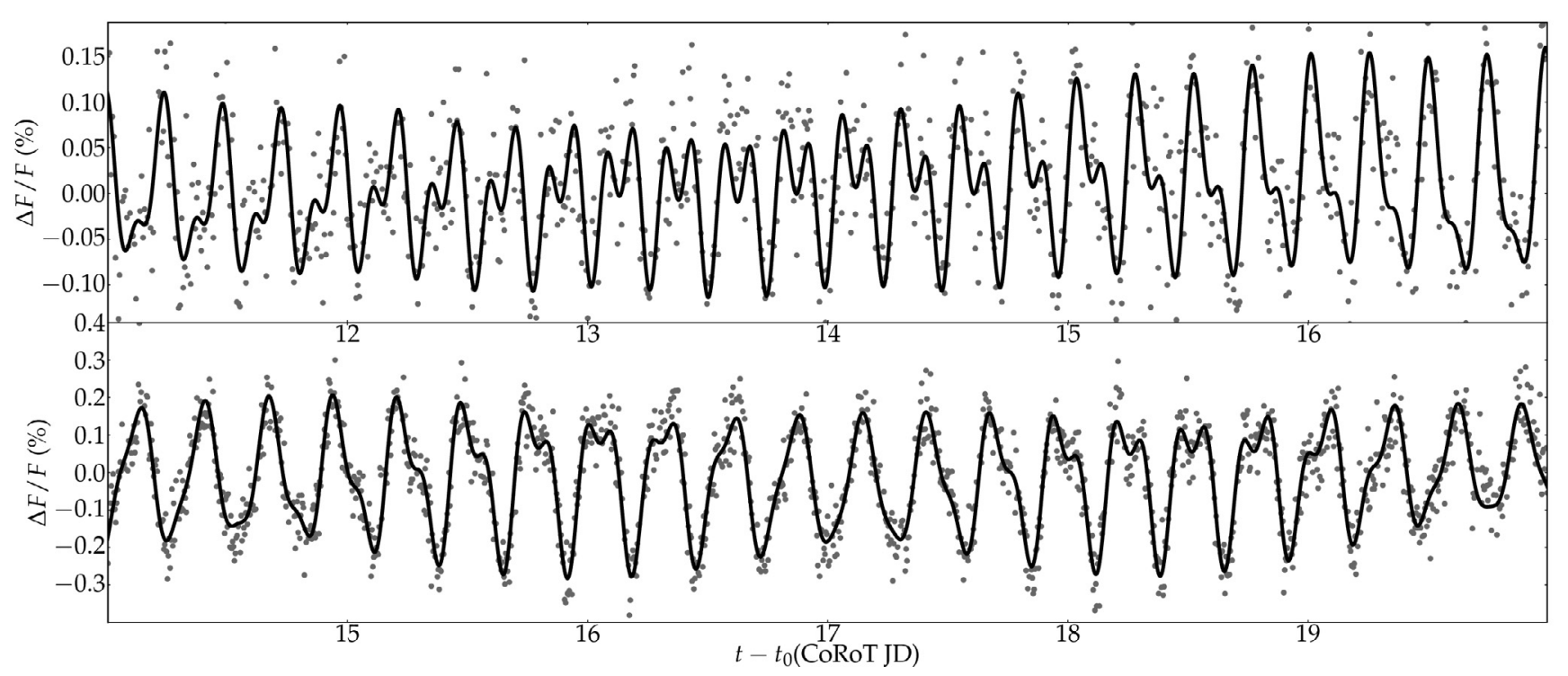

Fig. 17. Selection of $\beta$ Cep candidates showing strong hints of spot features in their light curves. (upper panel) CoRoT $102968526\left(t_{0}=\right.$ $2590.0450 \mathrm{CJD}$ ) has a dominant frequency of $f_{1} \approx 4.14 \mathrm{~d}^{-1}$ with a secondary peak at $f_{2} \approx 4.08 \mathrm{~d}^{-1}$. The black line is a fit using all noninstrumental frequencies (bottom panel) The light curve of CoRoT $102857565\left(t_{0}=2590.0499\right.$ CJD) was binned per 10 points for visibility reasons.

most interesting targets, and allow theoretical interpretation of the detected variability.

\section{Conclusions}

We constructed a frequency analysis pipeline and additional post-processing tools to efficiently analyse pulsators in CoRoT's exoplanet database. The method also includes a jump correction and detrending algorithm, as well as an automated search for combination frequencies and spacings among frequencies or periods. In the particular case of the Initial Run, many combination frequencies and spacings were found, but the total time span of $\sim 55 \mathrm{~d}$ was too short to draw firm conclusions on the frequency or period spacings of $g$ modes and to confront them with theoretical models. There were too few convincing candidates in the more massive $\beta$ Cep class to commence an interpretation in terms of nonlinear resonant coupling. However, our techniques revealed clear frequency spacings in high-frequency variables on the cool side of the SPB instability strip.

Most of the stars that seemed $\operatorname{good} \beta$ Cep candidates in terms of the values of the first three frequencies are to be refuted. This was to be expected, because the number of candidate $\beta$ Cep stars found by the CoRoT Variability Classifier (Debosscher et al. 2009) was anomalously high compared to the number of candidate SPBs. Only one star on the blue side of the SPB instability strip matches a typical $\beta$ Cep frequency spectrum. Four other stars were found to be $\delta \mathrm{Sct} / \beta \mathrm{Cep}$-like, but again were far off their theoretical location in the $\log T_{\text {eff }}-\log g$ diagram. Instead, we detected many different types of variable stars among the $\beta$ Cep candidate sample. Several turned out to be low-amplitude pulsators whose frequencies are spread over a wide range and which are located on the red side of the SPB 


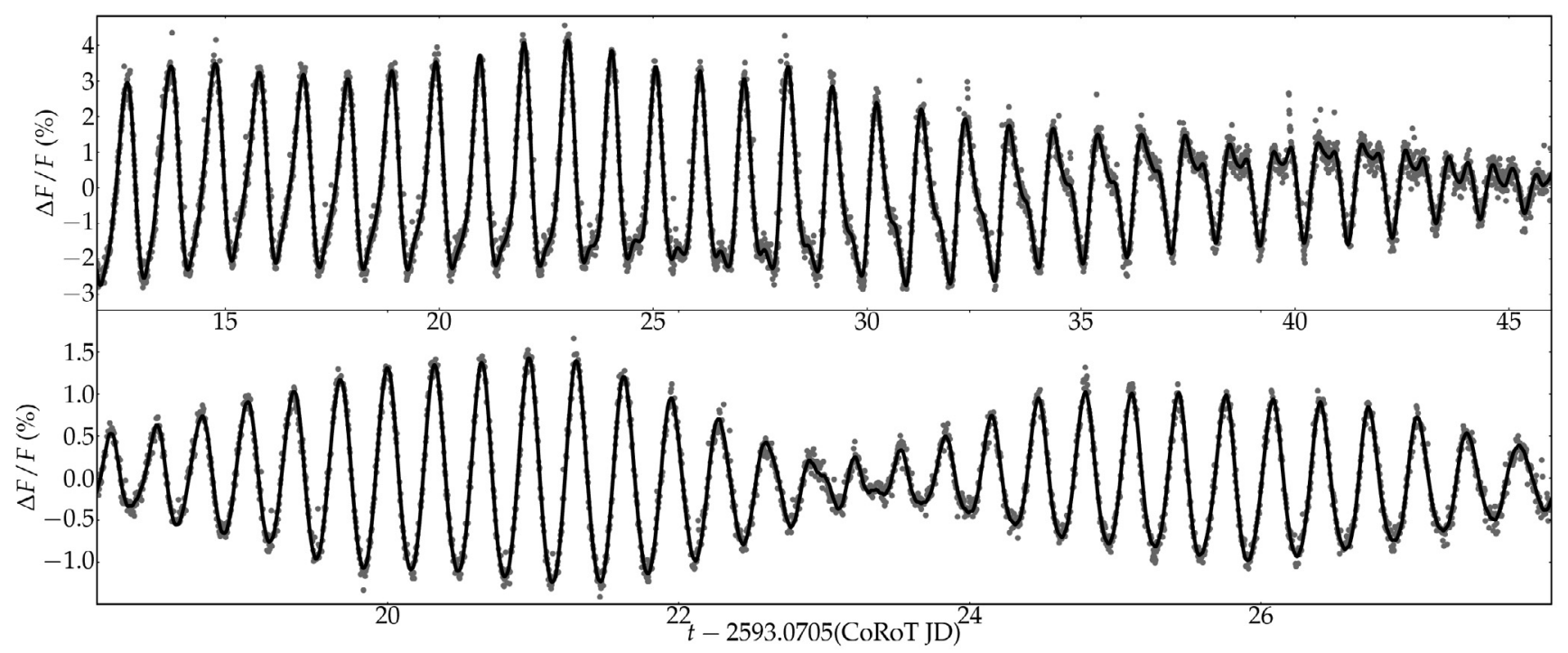

Fig. 18. CoRoT 102835515 (upper panel) and 102824452 (lower panel, light curve binned per 10 points) have a frequency spectrum where the power is concentrated in narrow bands at multiples of the dominant frequency. Combined with the peculiar shape of their light curves, they strongly resemble spotted stars, although the distinction from a simple beating pattern is not easy (bottom). The solid line is a fit, grey circles are data.

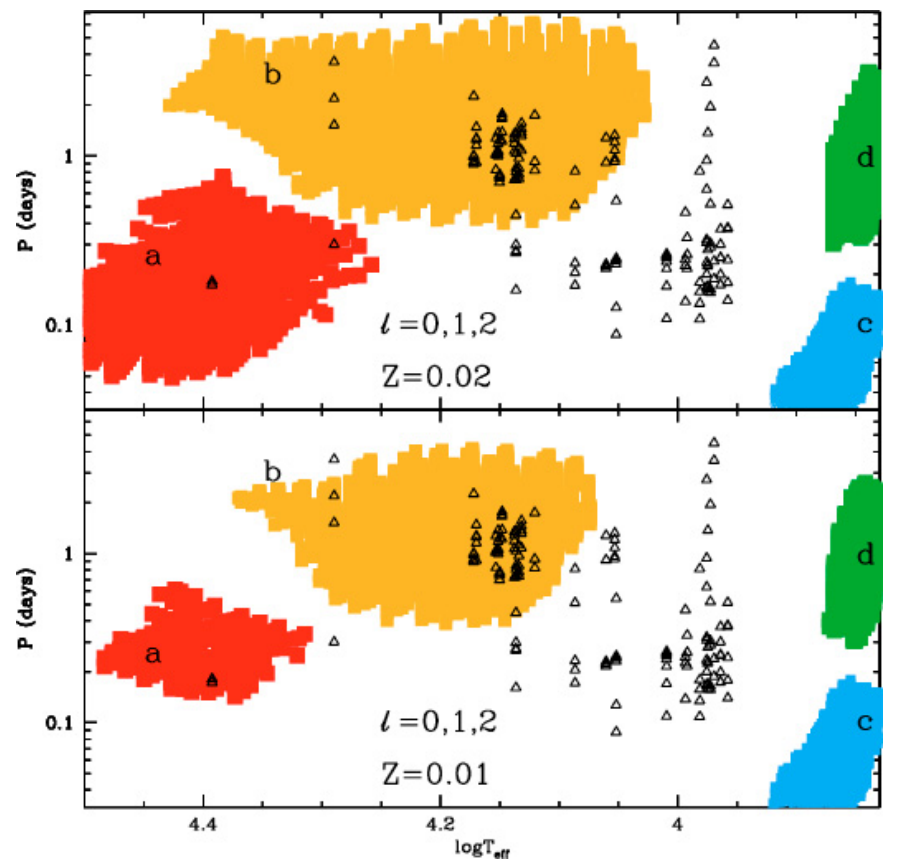

Fig. 19. Theoretical instability domains of $\ell=0,1,2$ modes represented in a $\log T_{\text {eff }}-\log P$ diagram for main-sequence models with $Z=0.02$ (upper panel) and $Z=0.01$ (lower panel). In each panel, the regions of unstable modes represent $\beta$ Cep (a), SPB (b), $\delta$ Sct (c), and $\gamma$ Dortype pulsations (d). The frequencies detected in the best SPB candidates (see text) and the candidate pulsators from Sect. 4.2 are shown as triangles.

instability strip, where no oscillations are predicted by theory. According to the theoretical computations by Townsend (2005) and Savonije (2005), mid-B type rapidly rotating pulsators with retrograde mixed $g$ modes could occur in a part of this region, exhibiting many of these properties. However, they could also simply be fast-rotating classical SPB or $\delta$ Sct stars, or Be pulsators. Future spectroscopic observations will allow us to distinguish between these possibilities, by supplying information on rotational velocities, abundances, and improved $\log g-\log T_{\text {eff }}$ values. Besides these interesting stars, also binary stars and a few suspectedly differentially rotating stars (with or without additional pulsations), were detected among the $\beta$ Cep candidates.

Similar conclusions apply to the sample of SPB candidates, although the number of good candidates is vastly greater for this class than for the $\beta$ Cep candidates. Interestingly, the highest amplitude SPB candidates all show two distinct signs of nonlinear behaviour: first, clear unexpected power excess in the region between 2 and $6 \mathrm{~d}^{-1}$, which seems to point to modes with finite lifetimes. These could originate from nonlinear nonresonant harmonic distortion, leading to time-dependent amplitudes and/or frequencies. Second, we identified combination frequencies, which are stable on the time scale of the measurements. These are likely to be caused by nonlinear resonant mode locking. On top of that, we also find some rich and low-amplitude SPB pulsators, with frequencies in a lower range than the similar stars in the $\beta$ Cep sample.

Our follow-up studies of new pulsating B stars in the CoRoT long runs, which typically last $150 \mathrm{~d}$, as well as future spectroscopy, will be very valuable in evaluating the new type of oscillations found in the Initial Run data as reported here in more detail.

Acknowledgements. The research leading to these results received funding from the European Research Council under the European Community's Seventh Framework Programme (FP7/2007-2013)/ERC grant agreement $N^{\circ} 227224$ (PROSPERITY), as well as from the Research Council of K.U. Leuven grant agreement GOA/2008/04 and from the Belgian PRODEX Office under contract C90309: CoRoT Data Exploitation. This article is based on observations made with the INT-WFC operated on the island of La Palma by the Isaac Newton Group in the Spanish Observatorio del Roque de los Muchachos of the Instituto de AstrofAysica de Canarias. This research has been partly supported by the AYA2006-15623-C02-02 of the MCINN. 


\section{References}

Aerts, C., \& Kolenberg, K. 2005, A\&A, 431, 615

Asplund, M., Grevesse, N., Sauval, A. J., Allende Prieto, C., \& Blomme, R. 2005, A\&A, 431, 693

Auvergne, M., Bodin, P., Boisnard, L., et al. 2009, A\&A, 506, 411

Badnell, N. R., Bautista, M. A., Butler, K., et al. 2005, MNRAS, 360, 458

Balona, L. A. 1994, MNRAS, 268, 119

Breger, M., Stich, J., Garrido, R., et al. 1993, A\&A, 271, 482

Buchler, J. R., Goupil, M.-J., \& Hansen, C. J. 1997, A\&A, 321, 159

Chapellier, E., Le Contel, D., Le Contel, J. M., Mathias, P., \& Valtier, J.-C. 2006, A\&A, 448, 697

Debosscher, J., Sarro, L. M., López, M., et al. 2009, A\&A, 506, 519

Degroote, P., Briquet, M., Catala, C., et al. 2009, A\&A, 506, 111

Dupret, M.-A., De Ridder, J., De Cat, P., et al. 2003, A\&A, 398, 677

Dziembowski, W. A., Moskalik, P., \& Pamyatnykh, A. A. 1993, MNRAS, 265, 588

Dziembowski, W. A., \& Pamiatnykh, A. A. 1993, MNRAS, 262, 204

Eyer, L., \& Bartholdi, P. 1999, A\&AS, 135, 1

Frescura, F. A. M., Engelbrecht, C. A., \& Frank, B. S. 2008, MNRAS, 388, 1693

Grigahcène, A., Dupret, M.-A., Gabriel, M., Garrido, R., \& Scuflaire, R. 2005, A\&A, 434, 1055

Gutiérrez-Soto, J., Neiner, C., \& Hubert, A. M. et al. 2008, CoAst, 157, 70

Handler, G., Shobbrook, R. R., Jerzykiewicz, M., et al. 2004, MNRAS, 347, 454

Handler, G., Jerzykiewicz, M., Rodríguez, E., et al. 2006, MNRAS, 365, 327

Huat, A.-L., Hubert, A.-M., Baudin, F., et al. 2009, 506, 95

Kawaler, S. D. 1988, in Advances in Helio- and Asteroseismology, ed. J. Christensen-Dalsgaard, \& S. Frandsen, IAU Symp., 123, 329

Koen, C. 2006, MNRAS, 371, 1390

Lanza, A. F., Rodono, M., \& Zappala, R. A. 1993, A\&A, 269, 351

Lomb, N. R. 1976, Ap\&SS, 39, 447
Miglio, A., Montalbán, J., \& Dupret, M.-A. 2007a, MNRAS, 375, L21

Miglio, A., Montalbán, J., \& Dupret, M.-A. 2007b, Commun. Asteroseismol., 151,48

Miglio, A., Montalbán, J., Noels, A., \& Eggenberger, P. 2008, MNRAS, 386, 1487

Montgomery, M. H., \& O’Donoghue, D. 1999, Delta Scuti Star Newsletter, 13, 28

Moon, T. T., \& Dworetsky, M. M. 1985, MNRAS, 217, 305

O'Donoghue, D. 1994, MNRAS, 270, 222

Saio, H., Cameron, C., Kuschnig, R., et al. 2007, ApJ, 654, 544

Samadi, R., Fialho, F., Costa, J. E. S., et al. 2006, ESA SP 1306, 317, corrected in [arXiv: astro-ph/0703354]

Savonije, G. J. 2005, A\&A, 443, 557

Scargle, J. D. 1982, ApJ, 263, 835

Scholz, G., Lehmann, H., Hildebrandt, G., Panov, K., \& Iliev, L. 1998, A\&A, 337,447

Schwarzenberg-Czerny, A. 2003, in Interplay of Periodic, Cyclic and Stochastic Variability in Selected Areas of the H-R Diagram, ed. C. Sterken, ASP Conf. Ser., 292, 383

Scuflaire, R., Montalbán, J., Théado, S., et al. 2008a, Ap\&SS, 316, 149

Scuflaire, R., Théado, S., Montalbán, J., et al. 2008b, Ap\&SS, 316, 83

Stankov, A., \& Handler, G. 2005, ApJS, 158, 193

Strassmeier, K. G., \& Bopp, B. W. 1992, A\&A, 259, 183

Tassoul, M. 1980, ApJS, 43, 469

Townsend, R. H. D. 2005, MNRAS, 364, 573

Vuille, F., O’Donoghue, D., Buckley, D. A. H., et al. 2000, MNRAS, 314, 689

Waelkens, C. 1991, A\&A, 246, 453

Weiss, W. W., Aerts, C., Aigrain, S., et al. 2004, in Stellar Structure and Habitable Planet Finding, ed. F. Favata, S. Aigrain, \& A. Wilson, ESA SP, 538,435

Zdravkov, T., \& Pamyatnykh, A. A. 2008, J. Phys. Conf. Ser., 118, 012079 
Appendix A: Additional figures \& tables

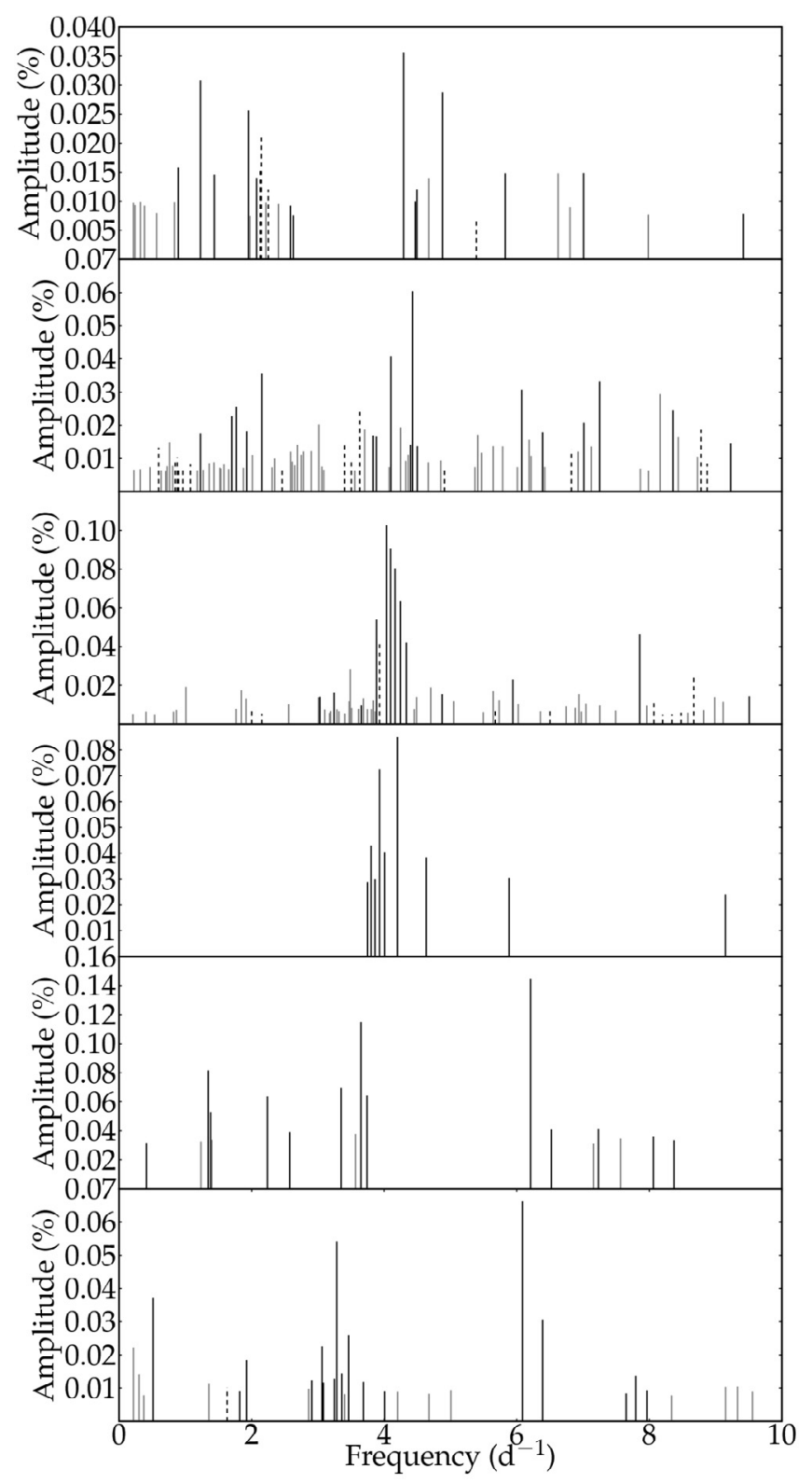

Fig. A.1. Schematic oscillation spectra of one group of pulsators of which the fundamental parameters $\log T_{\text {eff }}$ and $\log g$ place them in between the SPB and $\delta$ Sct instability strip (CoRoT 102861067, 102790063, 102729531, 102862454, 102790331, 102771057). These are all multiperiodic variables, showing complex variability in a broad frequency range (independent modes are depicted with black solid lines, harmonics as interrupted black lines, and candidate combination frequencies in grey).

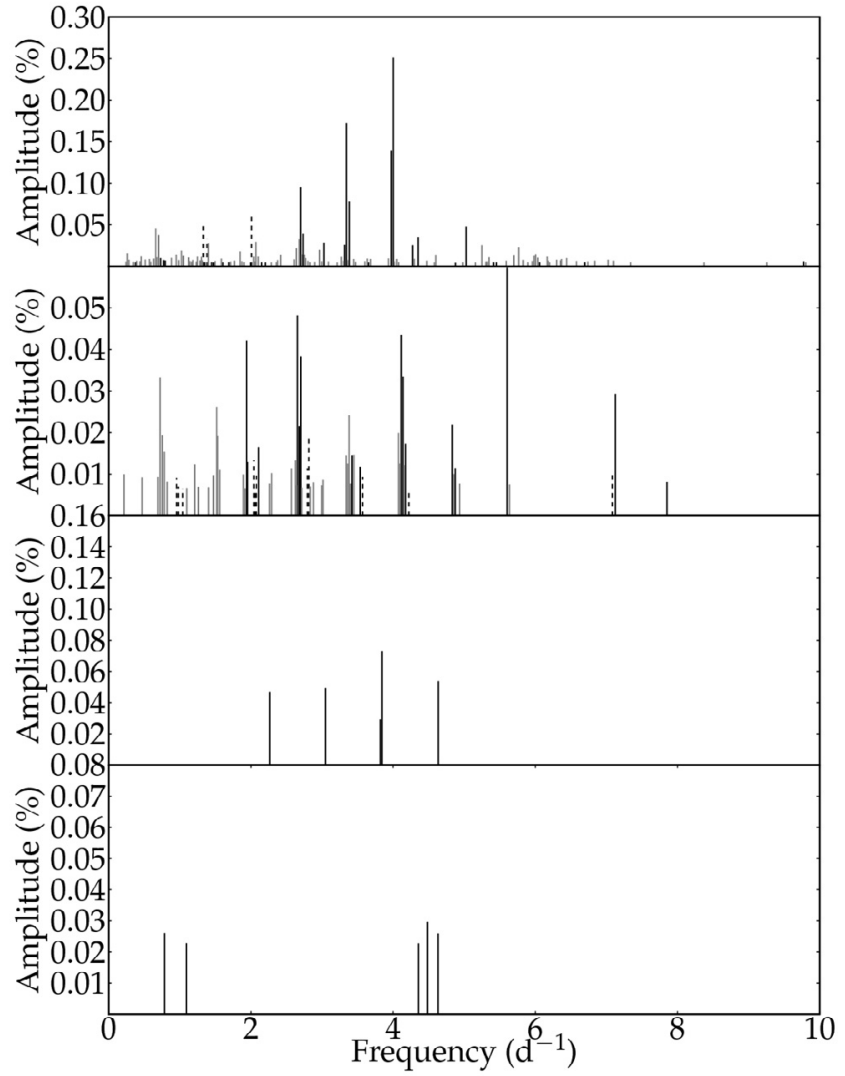

Fig. A.2. Sames as Fig. A.1, but for the stars showing a complex but structured frequency spectrum (CoRoT 102833548, 102848985, 102922479, 102850576).

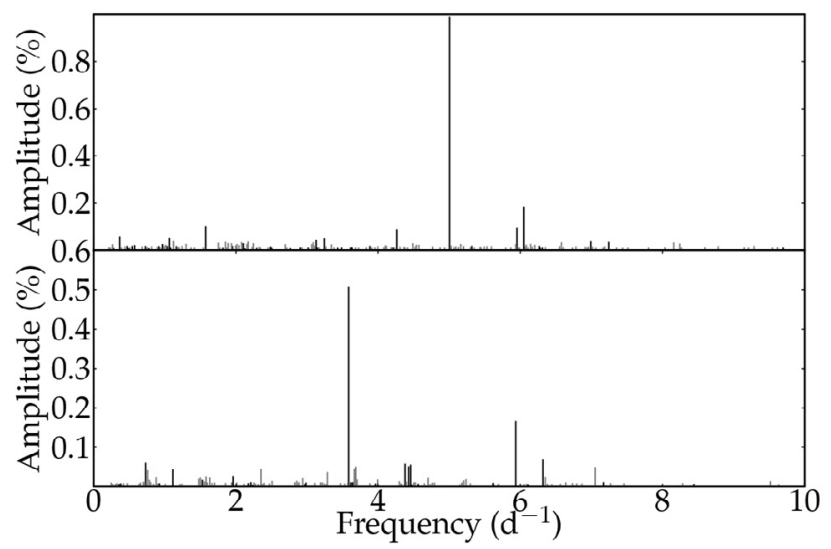

Fig. A.3. Same as Fig. A.1, but for two of the stars with a rich frequency spectrum, but having few large-amplitude pulsations (CoRoT 102850502, 102816758). 


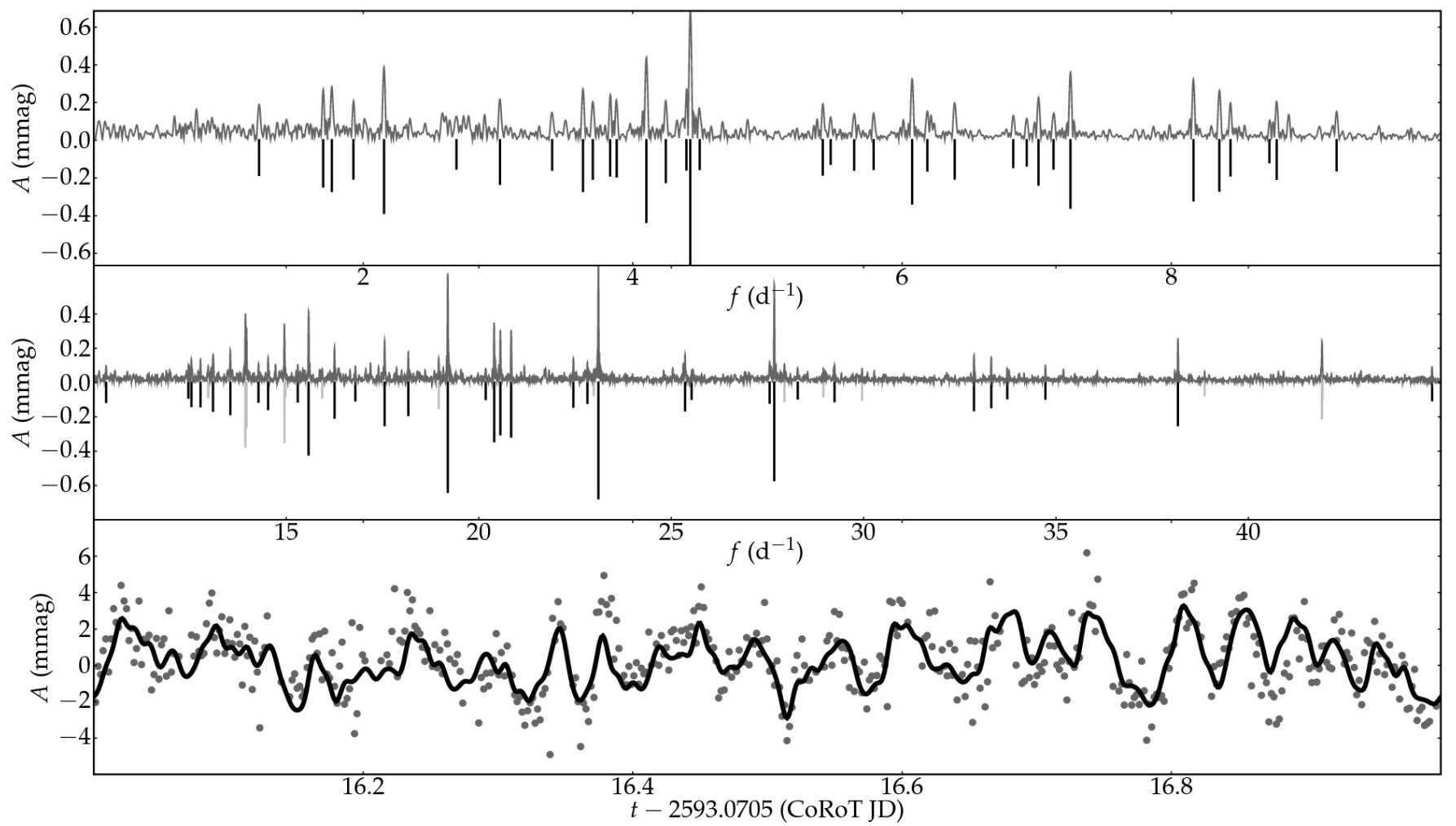

Fig. A.4. Same as Fig. A.5, but for the $\beta$ Cep candidate 102790063.

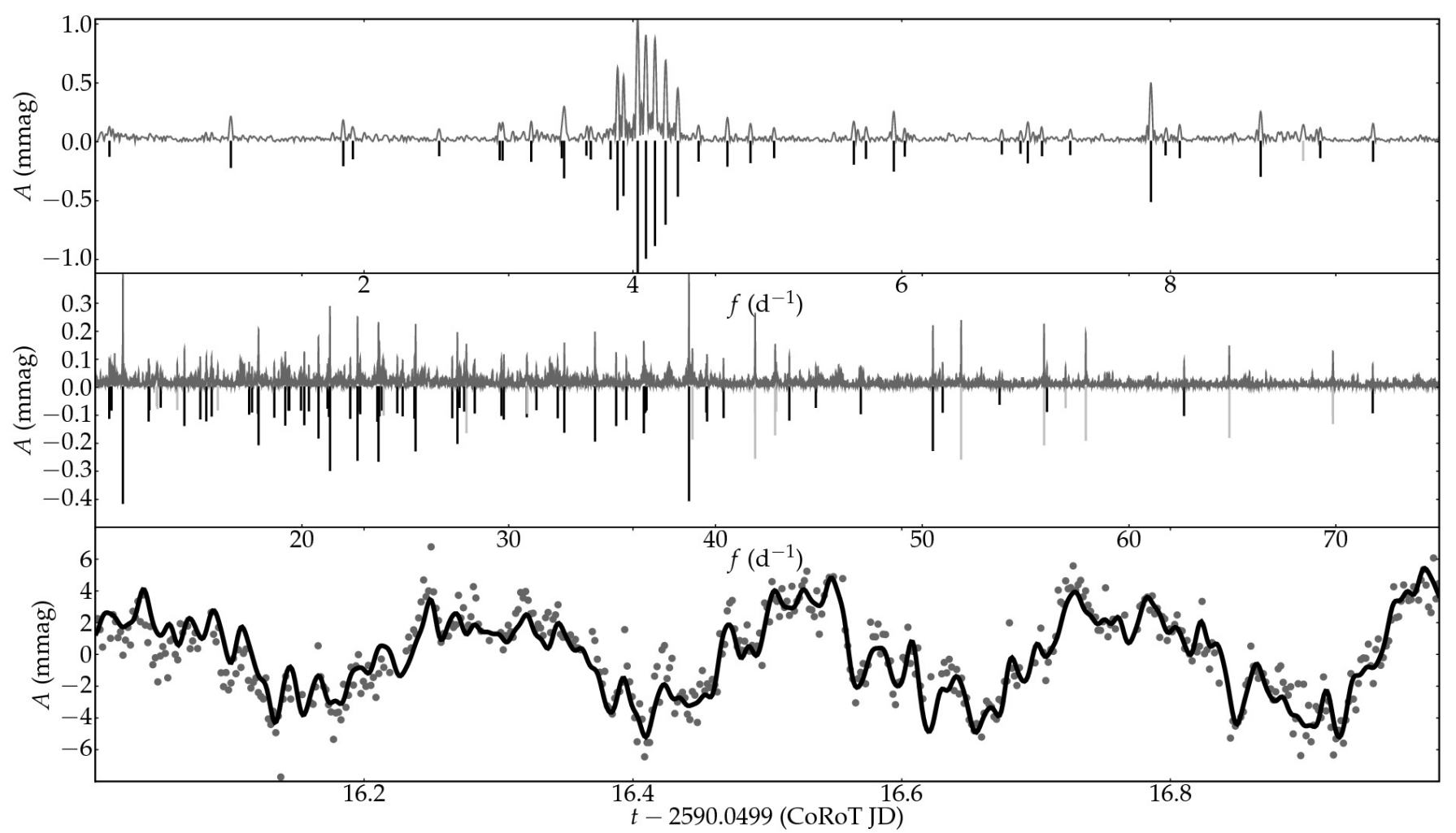

Fig. A.5. $\beta$ Cep candidate 102729531: (bottom panel) Zoom on day 17 of IRa01: on top of the clear $\sim 0.25$ d period, many more significant variations of the flux are noticable. (upper two panels) raw frequency spectrum (grey) with identified frequencies with $S N R>4$ (black lines) and orbital frequencies (grey lines, all harmonics and day aliases of 1 to 5 days). 


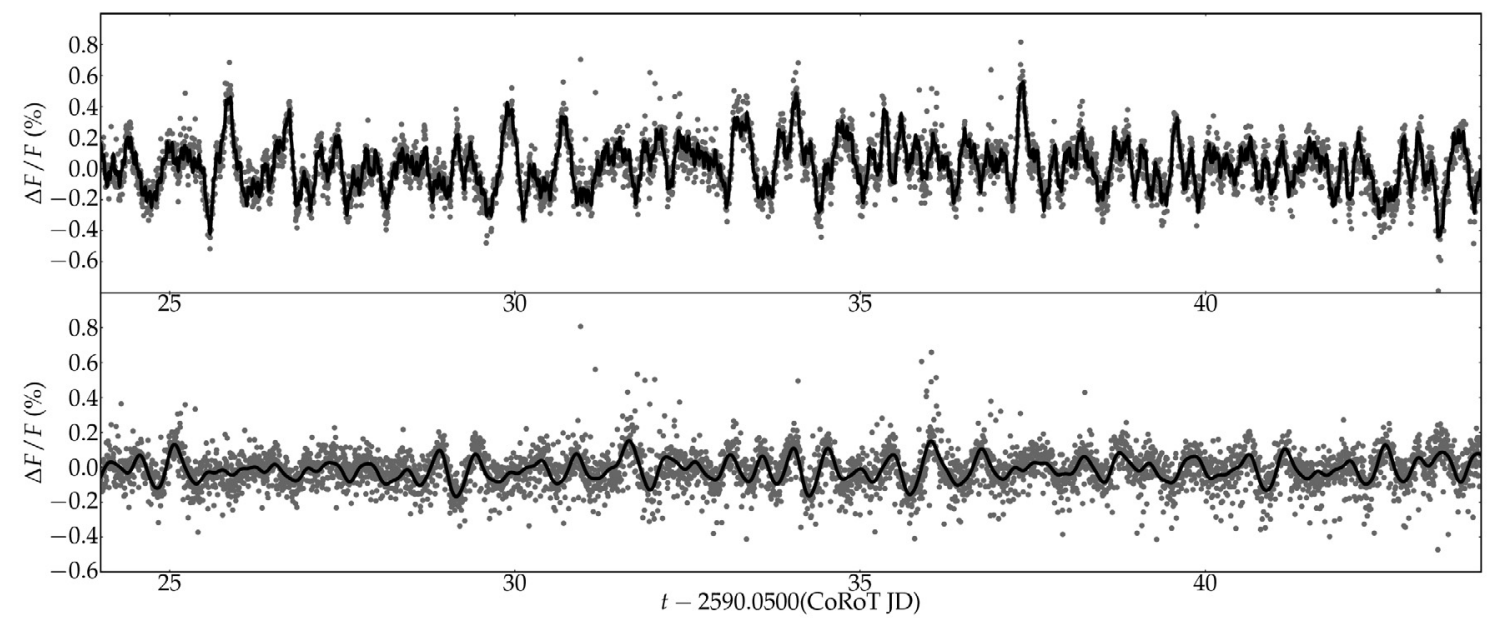

Fig. A.6. CoRoT 102872265 (upper panel) and 102832493 (lower panel) show a complex frequency spectrum with high peaks with frequencies above the typical SPB pulsation range. Moreover, the dense spectrum makes it difficult to seperate long, stable pulsations from beating patterns. The solid line is a fit, grey circles are the data.

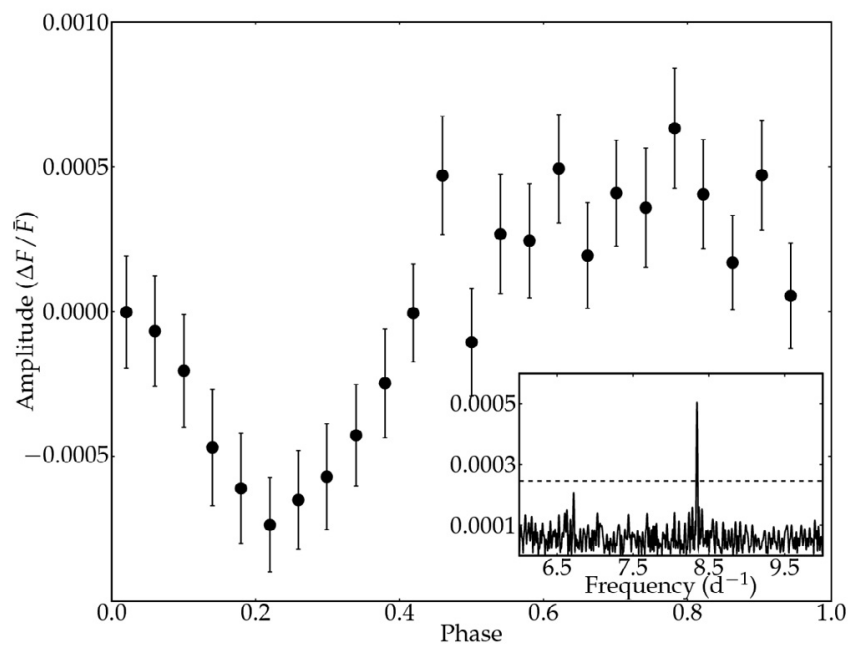

Fig. A.7. CoRoT 102808565: Binned phase diagram ( $1 \sigma$ errors on average), after removal of a nonlinear fit with 69 frequencies. Inset: excerpt from the residual Scargle periodogram.

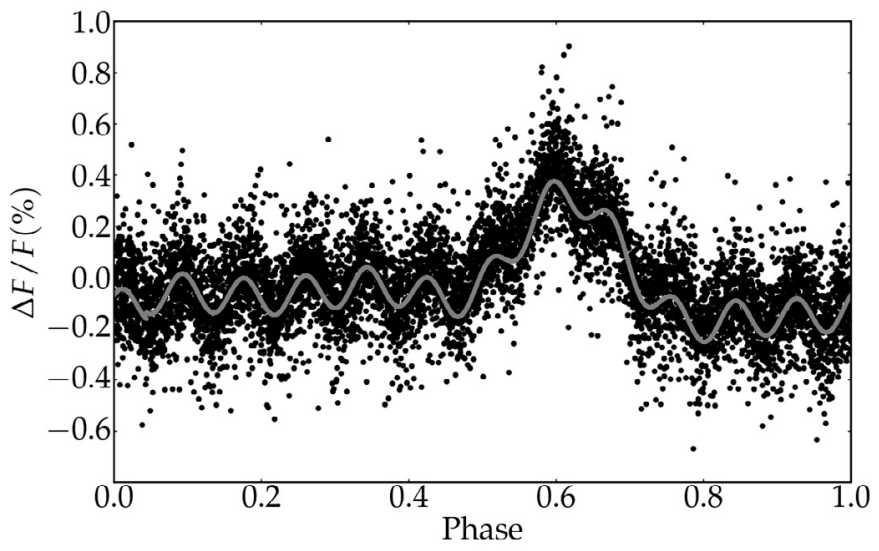

Fig. A.8. CoRoT 102762284: Phase diagram of the residual lightcurve after removal of the first two frequencies, which are both well approximated by sinusoidal variations. The diagram was folded with frequency $f=0.27812 \mathrm{~d}^{-1}$. A smaller period variation is noticable, as well as an outburst-like peak in the second half of the phase. 

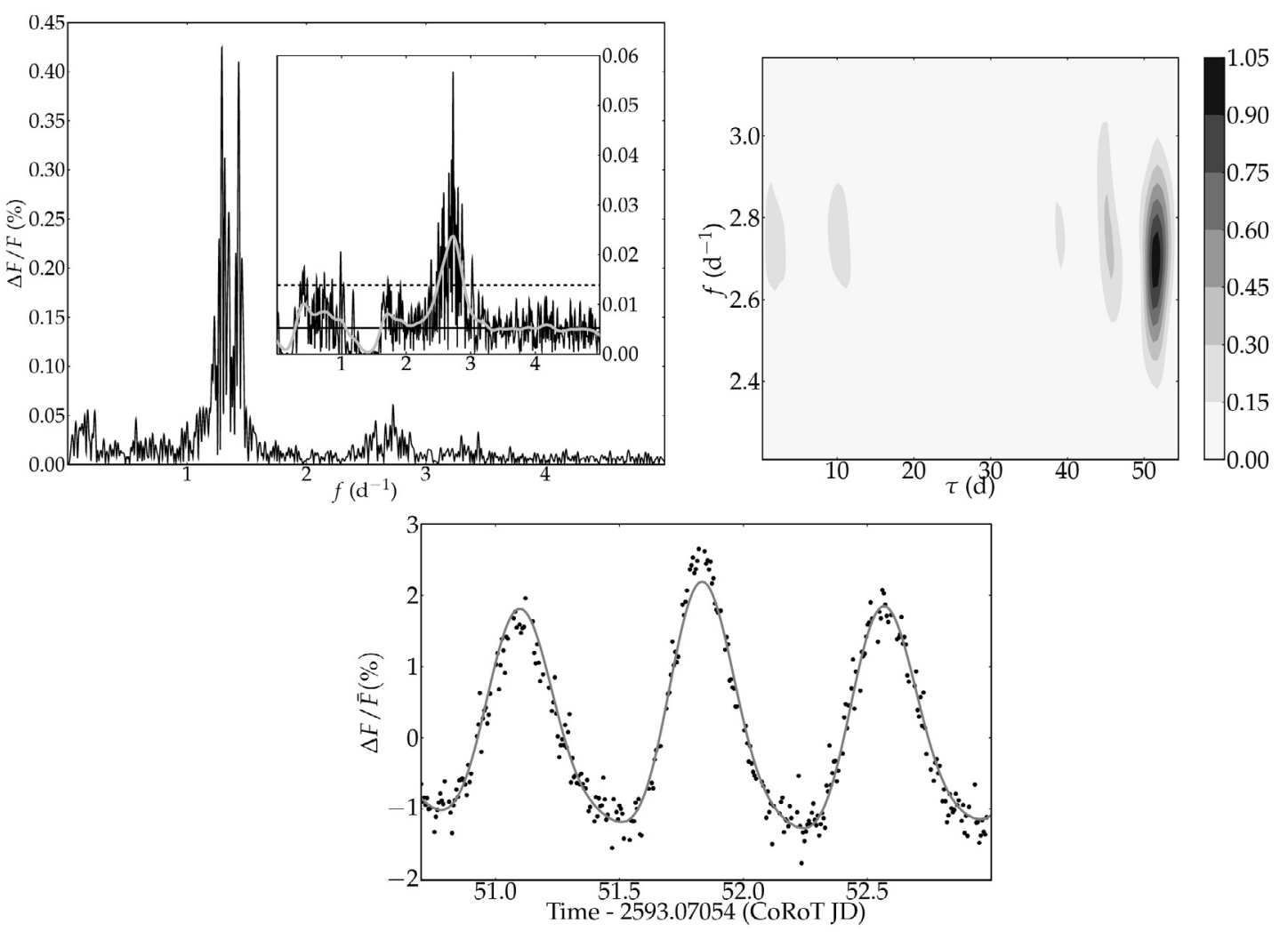

Fig. A.9. CoRoT 102943966: Original periodograms of SPB candidates showing residual power excess at frequencies above $\sim 1.5 \mathrm{~d}^{-1}$ (upper left). The insets are amplitude spectra of the residuals, after prewhitening all frequency in lower regions. The grey solid line is a smoothed version of the spectrum, horizontal lines denote theoretical noise level and 99\% confidence interval. upper right: Short time Fourier transformations of the residual light curves give the evolution of the (normalised) power in the periodogram over time since the start of the measurements $(\tau)$. This time-frequency analysis shows that the excess power is due to modes with a finite lifetime in the order of days. (lower panel:) excerpt from the light curve, showing the shape of the flux changes due to the pulsations.
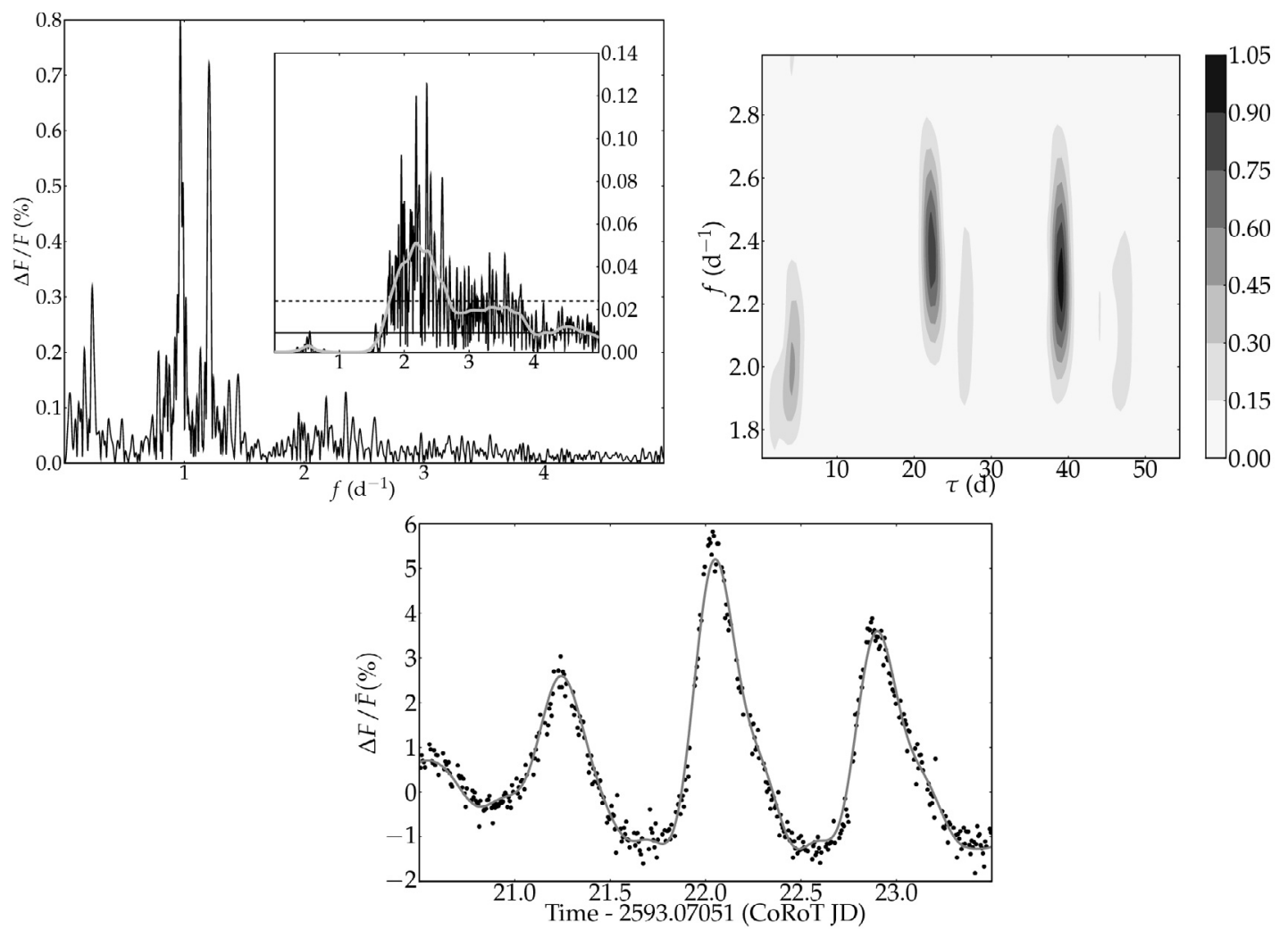

Fig. A.10. CoRoT 102855391: same as Fig. A.9. 

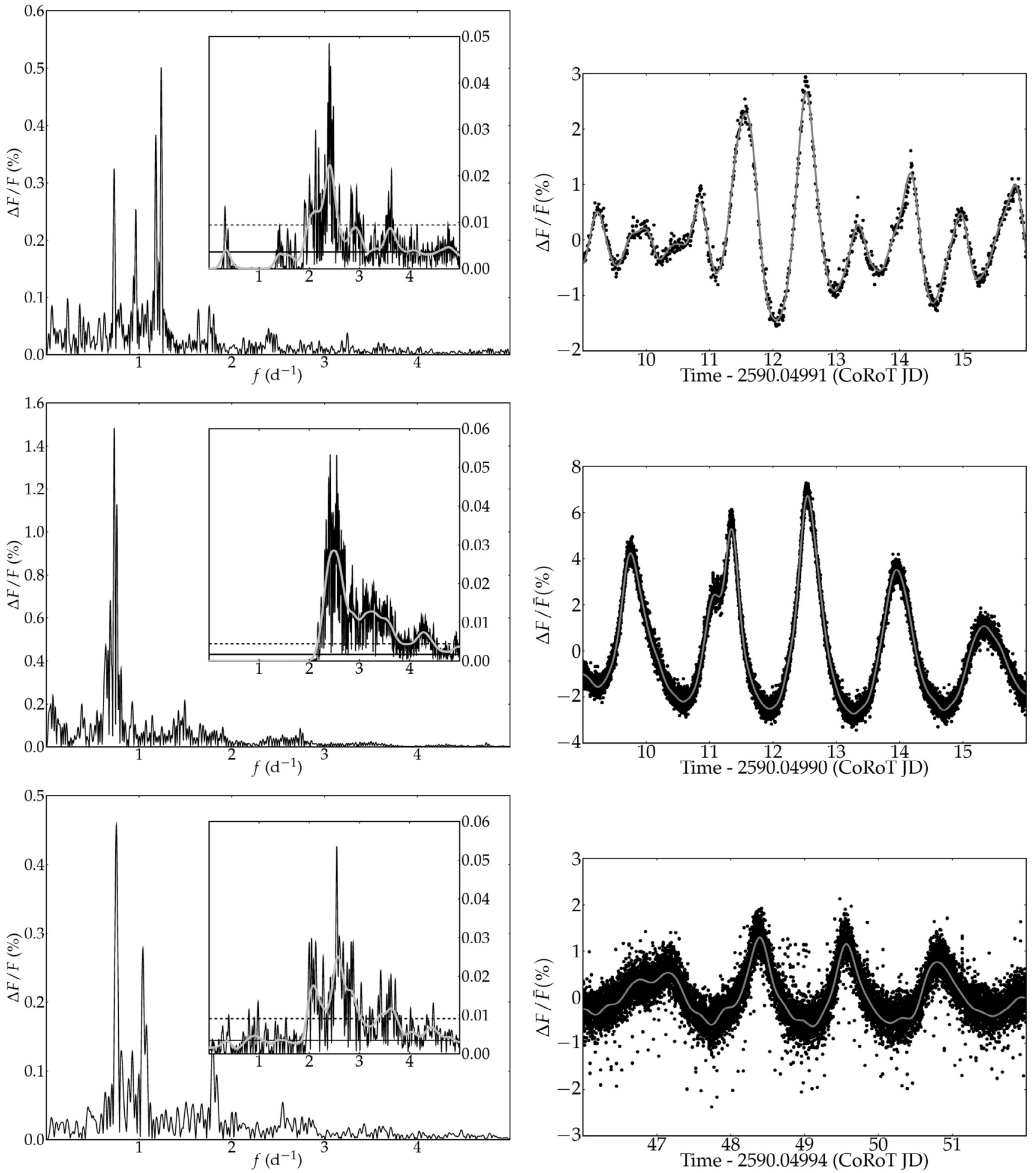

Fig. A.11. (left column) Original periodograms of SPB candidates showing residual power excess at frequencies above $\sim 1.5 \mathrm{~d}^{-1}$. The insets are amplitude spectra of the residuals, after prewhitening all frequency in lower regions. The grey solid line is a smoothed version of the spectrum, horizontal lines denote theoretical noise level and 99\% confidence interval. (right column) excerpt from the lightcurve, showing the shape of the flux changes due to the pulsations. (from top to bottom) CoRoT 102769848, 102754851 and 102848506. 

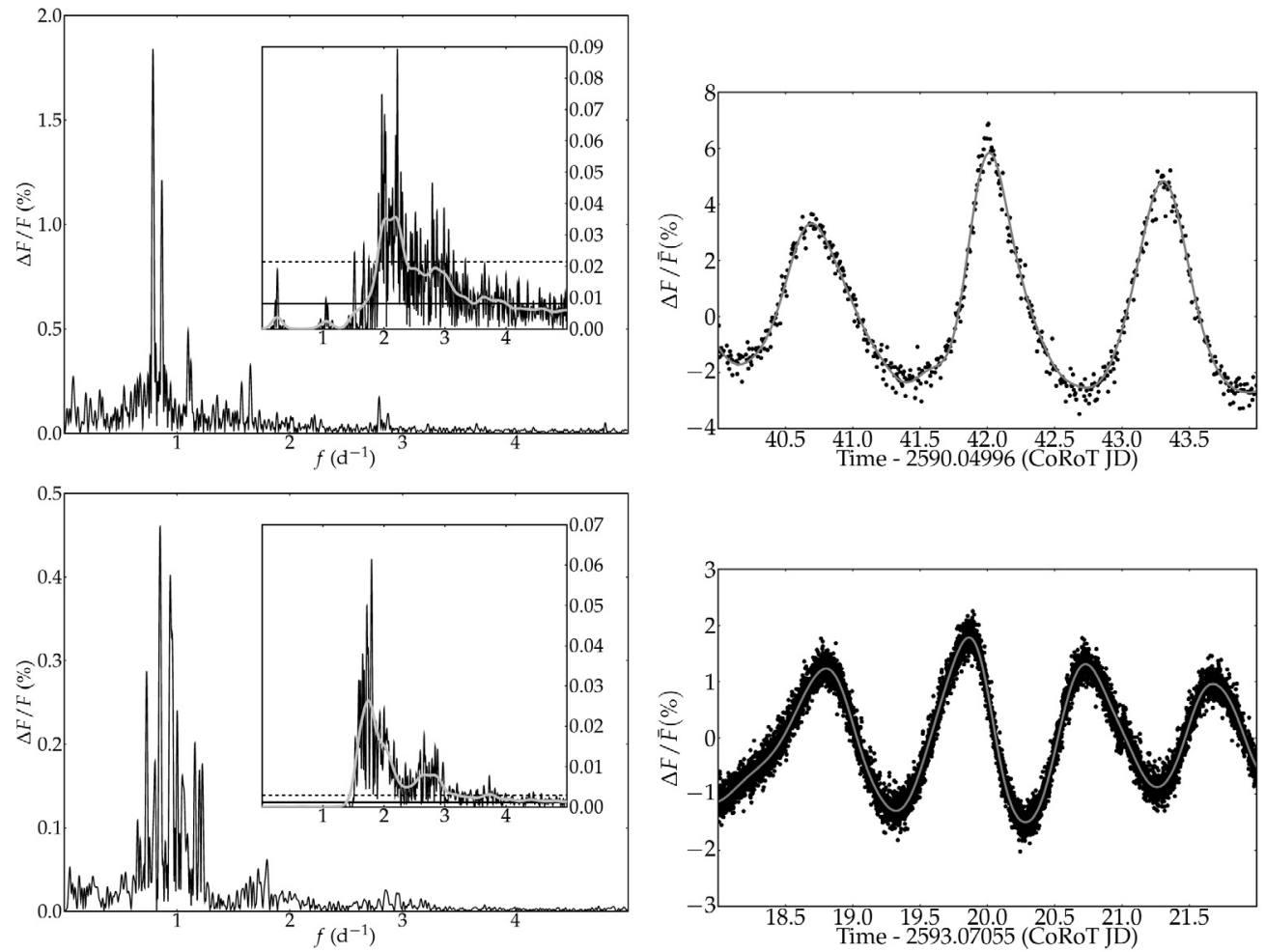

Fig. A.12. Same as Fig. A.11, but for CoRoT 102844894 and 102956197.

Table A.1. CoRoT 102804522 shows rich variability on varying timescales, but many of the frequencies can be connected through the same frequency spacing $\left(\epsilon=f_{2}-f_{1}-\Delta f\right)$.

\begin{tabular}{lcccr}
\hline \hline Type & $f_{1}\left(\mathrm{~d}^{-1}\right)$ & $\Delta f\left(\mathrm{~d}^{-1}\right)$ & $f_{2}\left(\mathrm{~d}^{-1}\right)$ & $\epsilon\left(\mathrm{d}^{-1}\right)$ \\
\hline Single & 1.0891 & 0.5320 & 1.6213 & 0.0002 \\
Single & 1.2350 & 0.5248 & 1.7595 & -0.0003 \\
Single & 1.4445 & 0.5248 & 1.9697 & 0.0004 \\
Single & 1.7595 & 0.5320 & 2.2914 & -0.0002 \\
Single & 1.8758 & 0.5320 & 2.4077 & -0.0002 \\
Single & 3.1081 & 0.5248 & 3.6325 & -0.0004 \\
Single & 3.6325 & 0.5248 & 4.1572 & -0.0001 \\
Single & 3.7455 & 0.5248 & 4.2703 & -0.0001 \\
Single & 3.8578 & 0.5320 & 4.3899 & 0.0001 \\
Single & 3.9212 & 0.5248 & 4.4458 & -0.0003 \\
Single & 4.3259 & 0.5248 & 4.8512 & 0.0004 \\
Single & 4.7765 & 0.5320 & 5.3088 & 0.0003 \\
Single & 9.0494 & 0.5248 & 9.5741 & -0.0001 \\
Triplet & 14.2073 & 0.5320 & 14.7395 & 0.0001 \\
& 14.7395 & 0.5320 & 15.2719 & 0.0004 \\
\hline
\end{tabular}

